J Geod.

DOI 10.1007/s00190-009-0304-7

\title{
Precise geodesy with the Very Long Baseline Array
}

\author{
Leonid Petrov • David Gordon · John Gipson • Dan MacMillan • \\ Chopo Ma • Ed Fomalont • R. Craig Walker • Claudia Carabajal
}

Received: 01 June, 2008/ Accepted: 26 January 2009

(C) Springer-Verlag 2009

\begin{abstract}
We report on a program of geodetic measurements between 1994 and 2007 which used the Very Long Baseline Array and up to 10 globally distributed antennas. One of the goals of this program was to monitor positions of the array at a 1 millimeter level of accuracy and to tie the VLBA into the International Terrestrial Reference Frame. We describe the analysis of these data and report several interesting geophysical results including measured station displacements due to crustal motion, earthquakes, and antenna tilt. In terms of both formal errors and observed scatter, these sessions are among the very best geodetic VLBI experiments.
\end{abstract}

Keywords VLBI · coordinate systems · plate tectonics . VLBA

L. Petrov,

ADNET Systems Inc./NASA GSFC, Code 610.2, Greenbelt, MD 20771 USA

E-mail: Leonid.Petrov@lpetrov.net

D. Gordon, J. Gipson, D. MacMillan

NVI Inc./NASA GSFC, Code 698, Greenbelt, MD 20771 USA

C. $\mathrm{Ma}$

NASA GSFC, Code 698, Greenbelt, MD 20771 USA

E. Fomalont,

National Radio Astronomy Observatory, 520 Edgemont Rd, Charlottesville, VA 22903-2475, USA

R. C. Walker

National Radio Astronomy Observatory, P. O. Box O, Socorro, NM 87801 USA.

C. Carabajal

Sigma Space Corporation/NASA GSFC, Code 698, Greenbelt, MD 20771 USA

Published online: 28 February 2009

\section{Introduction}

The method of very long baseline interferometry (VLBI), first proposed by Matveenko et al. (1965), is a technique of computing the cross-power spectrum of a signal from radio sources digitally recorded at two or more radiotelescopes equipped with independent frequency generators. This spectrum is used in a variety of applications. One of the many ways of utilizing information in the cross-power spectrum is to derive a group interferometric delay (Takahashi et al. 2000; Thompson et al. 2001). It was shown by Shapiro and Knight (1970) that group delays can be used for precise geodesy. The first dedicated geodetic experiment, on January 11, 1969, yielded 1 meter accuracy (Hinteregger et al. 1972). In the following decades VLBI technology flourished, sensitivities and accuracies were improved by several orders of magnitude, and arrays of dedicated antennas were built. Currently, VLBI activities for geodetic applications are coordinated by the International VLBI Service for Geodesy and Astrometry (IVS) (Schlüter and Behrend 2007).

Among dedicated VLBI arrays, the Very Long Baseline Array (VLBA) (Napier et al. 1994) of ten 25 meter parabolic antennas spread over the US territory (Figure 1) is undoubtedly the most productive. The VLBA is a versatile instrument used primarily for astrometry and astrophysical applications. All ten VLBA antennas have identical design (Figure 2). They have an altitudeazimuth mounting with a nominal antenna axis offset of $2132 \mathrm{~mm}$. Slewing rates are $1.5^{\circ} \mathrm{s}^{-1}$ in azimuth and $0.5^{\circ} \mathrm{s}^{-1}$ in elevation. Permanent GPS receivers are installed within 100 meters of 5 antennas, BR-VLBA, MKVLBA, NL-VLBA, PIETOWN, and SC-VLBA. 


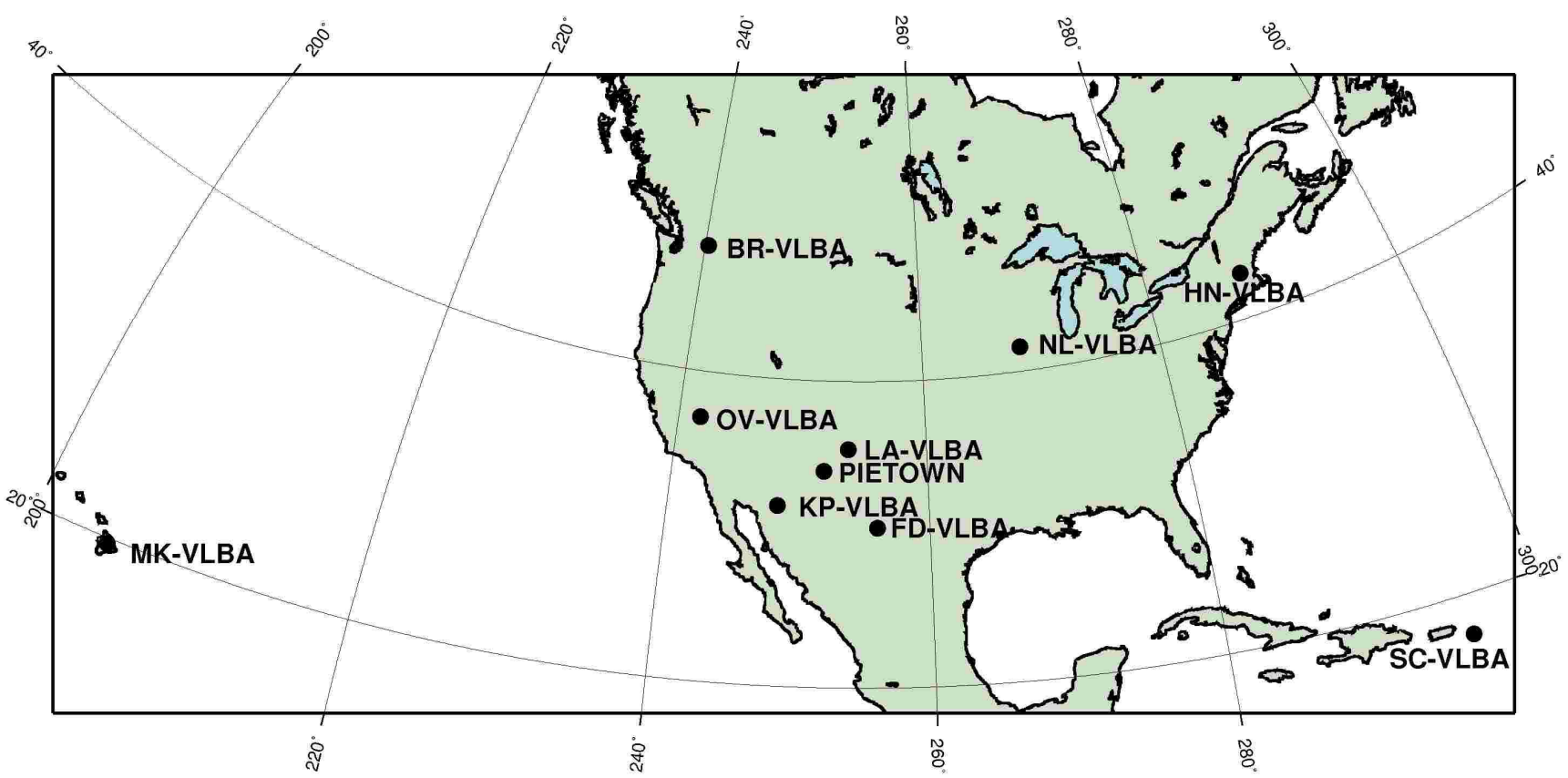

Fig. 1 Positions of the antennas of the Very Long Baseline Array

Phase referencing for detection of weak radio sources and for proper motion and parallax measurements are used in about half of all VLBA sessions. Accuracies on the order of 10 microarcseconds using source-tocalibrator separations of around one degree are achieved in the best current observations. Such accuracies need to be supported by the underlying geometric model and its input parameters, including the station and source catalogs and the Earth orientation parameters (EOP). A future goal is to improve on this accuracy by a factor of 2 or more. To achieve 10 microarcsecond accuracy on a $4000 \mathrm{~km}$ baseline, a delay accuracy after calibration of $0.2 \mathrm{~mm}$ or $0.6 \mathrm{ps}$ is required for any effects that cannot be reduced by integration. Phase referencing over a one degree source-to-calibrator separation reduces model errors by a factor of 57 , requiring the model parameters to be accurate to around $1 \mathrm{~cm}$. Higher accuracies are desired to deal with the cumulative effect of several model parameters, to meet future goals, or to allow larger source-to-calibrator separations.

Use of the Global Positioning System (GPS) can provide very high quality time series of site positions. Averaging these time series over several years can provide sub-mm estimates of the phase center positions of the GPS antennas, but this precision cannot be transferred to the reference points of the VLBI antennas for several reasons. First, measurements of the tie vector between the GPS phase center and the reference point of a radiotelescope introduce an additional uncertainty at a level of $3 \mathrm{~mm}$ or higher. Second, systematic errors of the GPS technique, such as phase center variations, multi-path, scale errors, and orbital errors, may cause biases in measurement of the phase center at a level of tens of $\mathrm{mm}$. Third, a nearby GPS receiver may not experience the same localized effects as the VLBI antenna, such as settling or tilting of the support structure. According to Ray and Altamimi (2005) (Table 4 in their paper), the root mean square (rms) of differences between coordinates of VLBI reference points derived from analysis of VLBI observations and from analysis of GPS observations plus ties measurements among 25 pairs of GPS/VLBI sites are $6 \mathrm{~mm}$ for the horizontal components and $13 \mathrm{~mm}$ for the vertical components after removal of the contribution of 14 Helmert transformation parameters fitted to the differences.

The best way for determining positions of the antenna reference points is to derive them directly from dedicated geodetic VLBI observations on the VLBA array. Uncertainties of better than $1 \mathrm{~mm}$ are easily achieved. Since the motion of these antenna reference points cannot be predicted precisely, geodetic observations need to be repeated on a regular basis in order to sustain that high precision.

The importance of precise position monitoring was recognized during the design of the VLBA and each antenna began to participate in geodetic VLBI observations soon after it was commissioned. Between July 1994 and August 2007, there were 132 dedicated 24 hour dual band S/X VLBI sessions under geodesy and absolute astrometry programs with a rate of 6-24 sessions per year. During each session, all ten VLBA antennas and up to 10 other geodetic VLBI stations par- 
ticipated. In this paper we present the geodetic results from this campaign. In section 2 we describe the goals of the observations, scheduling strategies and the hardware configuration. In section 3 we describe the algorithm for computing group delays from the output of the FX VLBA correlator and validation of the postcorrelator analysis procedure. The results and the error analysis are presented in sections 4 and 5 . Concluding remarks are given in section 6 .

\section{Observing sessions}

The primary goal of these geodetic VLBI observations was to derive an empirical mathematical model of the motions of the antenna reference points. The antenna reference point is the projection of antenna's moving axis (the elevation axis for altitude-azimuth mounts) to the fixed axis (the azimuthal axis for altitude-azimuth mounts). This mathematical model can be used for reduction of astronomical VLBA observations as well as for making inferences about the geophysical processes which cause this motion. A secondary goal was to estimate the precise absolute positions of many compact

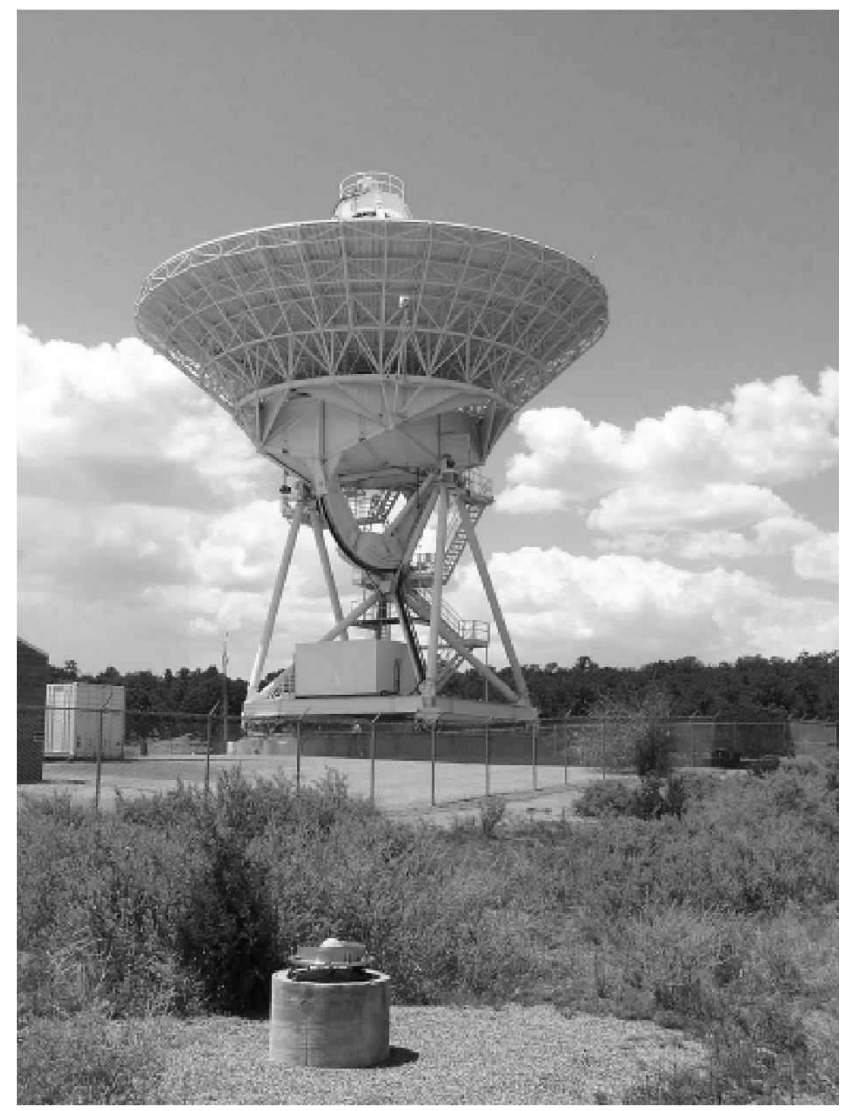

Fig. 2 The VLBA station PIETOWN is in the background. The permanent GPS receiver PIE1 is in the foreground. radio sources not previously observed under absolute astrometry programs, for use as phase referencing calibrators. Other goals, not discussed here, include monitoring a list of $\sim 400$ selected sources and producing time series of source coordinate estimates and images for improving the source position catalogue (A. Fey et al. (2009), paper in preparation) and for studying source structure changes (Piner et al. 2007; Kovalev et al. 2008).

The observing sessions were typically 24 hours long. The radio sources observed were distant active galactic nuclei at distances of a gigaparsec scale ${ }^{1}$ with continuum radio emission from regions of typically $0.1-10$ milliarcsecond in size.

VLBA geodetic observations use the dual frequency $\mathrm{S} / \mathrm{X}$ mode, observing simultaneously at $\mathrm{S}$ and $\mathrm{X}$ bands, centered around 2.3 and $8.6 \mathrm{GHz}$. This is enabled by a dichroic mirror permanently positioned over the $\mathrm{S}$ band receiver, reflecting higher frequency radiation towards a deployable reflector leading to the $\mathrm{X}$ band receiver. The system equivalent flux densities (SEFD) of VLBA antennas are in the range of 350-400 Jy when using the dual-frequency S/X system. From each receiver, four frequency channels $4 \mathrm{MHz}$ wide before April 1995 and $8 \mathrm{MHz}$ thereafter, were recorded over a large spanned bandwidth to provide precise measurements of group delays. The sequence of frequencies (called IF) was selected to minimize sidelobes in the delay resolution function and to reduce adverse effects of radio interference. The sequence was slightly adjusted over the 14 year period of observations in accordance with changes in the interference environment. The frequency sequence used in the session of 2007.08.01 is presented in Table 1.

Table 1 The range of frequencies in the observing session of 2007.08.01, in MHz.

\begin{tabular}{lll}
\hline & & \\
IF1 & 2232.99 & 2240.99 \\
IF2 & 2262.99 & 2270.99 \\
IF3 & 2352.99 & 2360.99 \\
IF4 & 2372.99 & 2380.99 \\
IF5 & 8405.99 & 8413.99 \\
IF6 & 8475.99 & 8483.99 \\
IF7 & 8790.99 & 8798.99 \\
IF8 & 8895.99 & 8903.99 \\
\hline
\end{tabular}

\subsection{Scheduling}

Among the 132 observing sessions, 97 can be characterized as global geodetic sessions and 35 as absolute astrometry sessions. They differ in scheduling strategy.

\footnotetext{
1 gigaparsec $\approx 3.2 \cdot 10^{9}$ light years $\approx 3 \cdot 10^{25} \mathrm{~m}$
} 
A wider list of 150-250 sources was observed in each astrometry session while a shorter list of $\sim 100$ objects was observed in each geodesy session.

\subsubsection{Scheduling of astrometric sessions}

Two lists of sources were observed in astrometry sessions: a list of 150-200 target sources and a list of 30-80 tropospheric calibrators. Selection of tropospheric calibrators was based on two criteria: a) the compactness at both $\mathrm{X}$ and $\mathrm{S}$ band, i.e. the ratio of the median correlated flux density at baselines longer than $5000 \mathrm{~km}$ to the median correlated flux density at baselines shorter than $900 \mathrm{~km}$, must be greater than 0.5 ; b) the correlated flux density at baselines longer than $5000 \mathrm{~km}$ must be greater than $0.4 \mathrm{Jy}$ at both $\mathrm{X}$ and $\mathrm{S}$ bands. These sources are frequently observed in other IVS geodetic programs.

Target sources were scheduled for $1-3$ scans, i.e. the period of time when antennas are on source and record the data, in a sequence that seeks to minimize slewing time needed for pointing all antennas to the next source. In the astrometry sessions, normally all antennas simultaneously observe the same object for the same duration. Scan durations were determined on the basis of the predicted correlated flux density and the SEFDs to get SNRs of the multi-band fringe amplitude greater than 20. The typical scan durations were $40-480 \mathrm{~s}$.

The sequence of target sources was interrupted every 1.5 hours, to observe $3-5$ tropospheric calibrators. The tropospheric calibrators were scheduled in such a way that at each station, at least one calibrator was observed in the ranges of $\left[7^{\circ}, 20^{\circ}\right]$ elevation, $\left[20^{\circ}, 50^{\circ}\right]$ elevation, and above $50^{\circ}$ elevation. The purpose of including tropospheric calibrators was a) to reliably estimate the zenith path delay of the neutral atmosphere in the least squares (LSQ) solution, and b) to link the positions of new or rarely observed target sources with those of frequently observed calibrators. Astrometric schedules were prepared with the NRAO software package SCHED. The efficiency of these schedules, i.e. the ratio of time on source to the total time of the observing session is typically $\sim 70 \%$.

\subsubsection{Scheduling of geodetic sessions}

The geodetic sessions involved $\sim 15-20$ geographically dispersed antennas with varying sensitivities. At any given time, few sources, if any, are visible by the entire network. Hence, in contrast to the astrometric sessions, at any instant different subsets of antennas will be observing different sources, and the integration time will vary from antenna to antenna in order to reach the required SNR. The minimum elevation angle for scheduled observations for all antennas in the geodetic VLBA sessions is set to 5 degrees.

These sessions were scheduled using the automatic scheduling mode of the SKED program. The scheduler sets up some general parameters that govern how the schedule is generated. The scheduler then generates all or part of the schedule and examines it for problems, such as prolonged gaps in the schedule when a station is idle. The scheduling parameters can be adjusted to minimize problems. The schedule can also be modified by adding or deleting observations. In its automatic mode, SKED generates a sequence of scans using the following algorithm:

1. SKED determines the current schedule time by looking at the latest time any station was scheduled, and taking the earliest of these times.

2. SKED updates the logical source-station visibility table for the current time. The rows of this table correspond to sources and the columns to stations. If a source is visible at a station, the location is marked as true, else it is false. Any row that has two or more true entries corresponds to a possible scan. This table is modified by the so-called "Major Options" which control which scans are actually considered. The important major options are: A) MinBetween. If a source has been observed more recently than MinBetween, it is marked as down at all stations. This prevents strong sources from being observed too frequently. B) MaxSlew. If the slew time for an antenna is longer than MaxSlew, the source is marked as down for the station. C) MinSubNetSize. If the number of stations which can see a given source is smaller than MinSubNetSize, the source is marked as down at all stations.

3. SKED scores scans based on their effect on Sky Coverage or Covariance optimizations. The user has the option of choosing which, with Sky Coverage the usual choice. A) For Sky Coverage, SKED calculates, for each station, the angular distance of the source from all previous scans over some time interval. It finds the minimum angular distance, and averages over all stations. This is the sources' skycoverage score. The larger the score, the larger the hole that will be filled by observing this source. B) For Covariance optimization, prior to scheduling, the scheduler specifies a set of parameters to be estimated, and a subset to optimize. For example, you might estimate atmosphere at each station, clocks at all but the reference station, and EOP, but you are only interested in optimizing EOP. Scans are ranked by the decrease in the sum of the formal errors of the optimized parameters when the con- 
sidered scan is added to the schedule. C) In either case, the top X\% of scans are kept for further consideration, where $\mathrm{X} \%$ is user settable, and is typically $30-50 \%$. The smaller $\mathrm{X} \%$ is, the more important the initial ranking.

4. Lastly the top X\% scans are ranked by a set of "Minor Options". There are 15 Minor Options, each corresponding to some possible desirable feature of the scan. For each scan, SKED calculates the weighted sum of the minor options in use. The scan with the highest overall score is scheduled. A description of all of the Minor Options and how the score is calculated is beyond the scope of this paper. The Minor Options typically used for scheduling geodetic VLBA experiments, and their effect on the scan selection follows. A) EndScan prefers scans which end soonest. B) NumObs prefers scans with more observations, i.e., with more stations. C) StatWt prefers scans involving certain stations. This is a way of increasing the number of observations at weak stations, or stations that are poorly connected to the network. D) StatIdle prefers scans which involve stations which have been idle. This reduces gaps in the schedule. E) Astrometric and F) SrcEvn modes are discussed below.

When SKED is done scheduling a scan, it checks to see if there is more time left, in which case it returns to Step 1. If not, it returns control to the scheduler.

Geodetic VLBA experiments have two goals absent from other geodetic VLBI sessions: 1) The inclusion of "requested" sources for which precise positions have been requested by the astronomical community; and 2) The desire to image all (or most) of the sources in each experiment. These lead to the development of Astrometric mode and SrcEvn modes in SKED. In Astrometric mode the user specifies minimum and maximum observing targets for a list of sources. SKED preferentially selects scans involving sources which are below their targets, and discriminates against scans involving sources which are above their targets. SrcEvn mode was introduced because SKED has a tendency to select strong sources with good mutual visibility. If SrcEvn mode is turned on, SKED will preferentially schedule sources that are under-observed compared to their peers. This is one way of ensuring that weak sources, or sources with low mutual visibility, are observed a sufficient number of times so that they can be imaged. The efficiency of geodetic schedules is typically $45-60 \%$.

\subsection{Session statistics}

The distribution of sessions over time is presented in Table 2. In each session 7,000-34,000 pairs of S/X group delays were evaluated, for a total of 1,737947 values. The ten VLBA stations and 20 other non-VLBA stations took part in the observing campaign, with from 9 to 20 stations in each session. The frequency of station participation in sessions is shown in Table 3. Among 4412 observed sources, at least two usable $\mathrm{S} / \mathrm{X}$ pairs of group delays were determined for 3090 objects.

Table 2 Statistics of VLBA sessions

\begin{tabular}{ccc}
\hline Year & $\begin{array}{r}\text { \# geodetic } \\
\text { sessions }\end{array}$ & $\begin{array}{r}\text { \# astrometric } \\
\text { sessions }\end{array}$ \\
\hline 1994 & 3 & 1 \\
1995 & 12 & 2 \\
1996 & 16 & 8 \\
1997 & 6 & 5 \\
1998 & 6 & 0 \\
1999 & 6 & 0 \\
2000 & 6 & 0 \\
2001 & 6 & 0 \\
2002 & 6 & 2 \\
2003 & 6 & 0 \\
2004 & 6 & 4 \\
2005 & 6 & 7 \\
2006 & 7 & 5 \\
2007 & 5 & 1 \\
\hline
\end{tabular}

\section{Correlation and post-correlation analysis of observations}

Observations at individual stations were recorded on magnetic tapes or, since 2007, on Mark 5 disc packs. Cross-correlation of the raw data was performed on the VLBA correlator (Benson 1995; Walker 1995), in Socorro, N.M., USA. The correlator uses the GSFC program Calc and the station clock offsets with respect to UTC measured with GPS clocks to compute theoretical delays to each station. Each station's bit stream is offset by these delays during the correlation. The resultant correlator output is the amplitudes and residual phases as functions of time (visibility points) for each station, referenced to a common point that lies close to, but not necessarily coincident with the geocenter.

Most geodetic VLBI experiments are correlated using Mark 4 correlators (Whitney et al. 2004). Their output is processed using the Fourfit program developed at MIT Haystack Observatory. Since this program cannot handle the output from the FX correlators, we used the AIPS software package (Greisen 2003) for further processing. 
Table 3 Statistics of observing session per station. (1) IVS station name; (2) geocentric latitude; (3) longitude, positive towards east; (4) Number of observing sessions under geodesy and astrometry with the VLBA array.

\begin{tabular}{lrrr}
\hline \multicolumn{1}{c}{$(1)$} & $(2)$ & $(3)$ & $(4)$ \\
\hline PIETOWN & +34.122 & 251.880 & 132 \\
LA-VLBA & +35.592 & 253.754 & 130 \\
KP-VLBA & +31.783 & 248.387 & 129 \\
BR-VLBA & +47.939 & 240.316 & 128 \\
OV-VLBA & +37.046 & 241.722 & 128 \\
FD-VLBA & +30.466 & 256.055 & 126 \\
HN-VLBA & +42.741 & 288.013 & 126 \\
MK-VLBA & +19.679 & 204.544 & 124 \\
NL-VLBA & +41.580 & 268.425 & 123 \\
SC-VLBA & +17.645 & 295.416 & 117 \\
WESTFORD & +42.431 & 288.511 & 59 \\
KOKEE & +21.992 & 200.334 & 57 \\
GILCREEK & +64.830 & 212.502 & 56 \\
ONSALA60 & +57.220 & 11.925 & 50 \\
WETTZELL & +48.954 & 12.877 & 50 \\
MEDICINA & +44.328 & 11.646 & 46 \\
NYALES20 & +78.856 & 11.869 & 38 \\
TSUKUB32 & +35.922 & 140.087 & 31 \\
HARTRAO & -25.738 & 27.685 & 28 \\
GGAO7108 & +38.833 & 283.173 & 24 \\
TIGOCONC & -36.658 & 286.974 & 21 \\
NRAO20 & +38.245 & 280.160 & 20 \\
MATERA & +40.459 & 16.704 & 19 \\
HOBART26 & -42.611 & 147.440 & 6 \\
KASHIM34 & +35.772 & 140.657 & 6 \\
ALGOPARK & +45.763 & 281.927 & 5 \\
NOTO & +36.691 & 14.989 & 4 \\
ZELENCHK & +43.595 & 41.565 & 3 \\
SVETLOE & +60.367 & 29.781 & 2 \\
URUMQI & +43.279 & 87.178 & 1 \\
\hline & & &
\end{tabular}

\subsection{Using AIPS to process geodesy experiments}

Additional processing is required to evaluate the geodetic/astrometric VLBI observables of group delays and phase delay rates. The initial calibrations are:

1. Small amplitude corrections for the correlator statistics are applied while reading the raw data into an AIPS data base.

2. The reference point of each IF channel is moved from the lower frequency edge to the center frequency of the channel, along with an adjustment to the frequency in the AIPS data base. This reduces edge effects, resulting in a small improvement in determination of the group delays.

3. Bad antenna and frequency channels are flagged out, as necessary.

4. At the VLBA stations, relative phase and delay offsets are applied to the visibility points using measured phase calibration tone phases. For both VLBA and non-VLBA stations, manual phase offsets are applied. ${ }^{2}$ The phase offsets are determined by fringe fitting a reference scan on a compact radio source to determine the relative instrumental phase and residual group delay for each individual IF. These phases are removed from the entire data set, equivalent to setting the single band and multiband residual delays to zero at the scan used for the calibration.

The heart of the reduction process is the fringe fitting of the data using AIPS task FRING. Data for each scan, baseline, frequency band and IF channel are processed separately, and the following parameters are determined: the average phase at some fiducial time near the center of the scan; the average phase rate with time (fringe rate); and the average phase rate with frequency (single-band delay) by finding the maximum of the 2D Fourier-transform of visibility data (Takahashi et al. 2000) and subsequent LSQ fit. An SNR cutoff of about 3 is generally used in order to omit noisy solutions for relatively weak sources ${ }^{3}$.

For those observations in which all of the IF channels have a detection, an AIPS program called MBDLY computes the average phase for the reference frequency and the average phase slope with frequency (so-called multi-band delay or group delay) that best fits the individual IF phases obtained from FRING. The individual IF solutions for the single-band delay and the fringe rate are averaged over all IFs. Checks of the quality of the group delays are obtained by the spread of the individual single-band delays, the fringe rates, and the phase scatter between each measured IF phase versus the best-fit group delay. Observations with large deviations are flagged as low quality and generally are not used in the analysis.

The results from FRING and MBDLY give the phase, single-band delay, fringe-rate, and group delay for a fiducial time near the middle of each scan for each baseline and each frequency band. These quantities represent the residual values with respect to the correlator model for the observation. When these data are added to that of the correlator model, the results become the total phase delay, total single-band delay, total fringerate and total group delay, respectively. These total values are independent of the correlator model.

The correlator model is attached to the correlator output. For each source and each antenna, it is represented by a six-order polynomial for every two minute interval, so that its value can be determined at any time with rounding errors below 0.1 ps. It contains

2 The non-VLBA stations have phase calibration systems, but their phases could not be captured in real time, nor extracted during correlation as is done on the Mark 4 correlators.

3 The AIPS cookbook can be found on the Web at http://www. aips.nrao.edu/cook.html 
three parts: the geometric delay based on the a priori source position, antenna locations, and the Earth orientation parameters; an a priori atmospheric delay; and the clock offset with respect to UTC determined by GPS at each individual station. The time-tag associated with the correlator model and the residual parameters is earth-center oriented. That is, the parameters are referenced to the time when the wavefront intercepts a fiducial point chosen at the coordinate system origin in order to facilitate the correlation process. The total quantities are then determined by adding the baseline residual parameters to the correlator model difference for the appropriate antenna-pair, interpolated to the scan reference time.

The total observables are continuous functions of time. Further geodetic and astrometric analysis requires discrete values of observable quantities, one per scan and per baseline, thereafter called observations, with time-tags associated with the arrival of the wavefront at the reference antenna of the baseline. These observables can differ by as much as $20 \mathrm{~ms}$ from the quantities with Earth-centered time-tags. AIPS task CL2HF is used to combine the correlator models and residuals at two stations and compute the observables with the reference antenna time-tags. For convenience, the time-tags are chosen to be on an integer second, and a common timetag is set to all observations in a scan. CL2HF performs this transformation, computes the fringe amplitude SNRs, delay, and rate uncertainties. CL2HF writes out an "HF" extension AIPS file which contains the total quantities as well as many other derived quantities needed for further analysis. Finally, the AIPS task HF2SV converts the data in the HF extension file to a binary form that is consistent with Mark 3 correlator output.

3.2 Validation of the post-correlation analysis procedure

For the first few years, the VLBA/AIPS processed sessions were freely mixed with Mark 4/Fourfit processed sessions, with few noticeable effects. However, two discrepancies were noticed between results from the two data sets. 1) The horizontal position of the ONSALA station shifted by approximately $3 \mathrm{~mm}$ between the two sets of data and 2) scatter of source position series for southern sources differed at a level of $0.2-1.0$ mas. The shift in ONSALA's position was found to be the result of a strong azimuthal dependence of instrumental delay in the cable, not seen at other sites. It showed up because measured phase calibration was not used for ONSALA in the VLBA/AIPS processing. The source statistics difference was found to be due to an incorrect accounting in program CL2HF of the total number of bits read from the station tapes at the VLBA correlator. When this was corrected, the "southern source" problem disappeared.

A direct comparison of delays and rates processed by the AIPS software package versus the Haystack Fourfit software package was strongly desired. To make such a comparison, the tapes from 8 stations in the rdv22 VLBA session (2000 July 6-7) were saved and sent to Haystack Observatory, where they were correlated on the Mark 4 correlator and fringed using the program Fourfit. To minimize the differences in processing, a single set of phase calibration phases was used in both the AIPS and Fourfit processing. Two databases were made of the same baselines processed through the two independent systems, with matching time tags. The regular Calc/Solve analysis was then performed on each to eliminate any bad data points. The observed delays and rates were differenced and tabulated by baseline. There were constant offsets for each baseline due to differences in single band calibration, and differences in $2 \pi$ ambiguity shifts. Such delay differences get absorbed into the clock adjustments and do not affect the geodetic or astrometric results. After removal of these constant differences, weighted root mean square (wrms) differences at X-band were computed by baseline. These are given in Table 4 . The wrms differences range from as little as $2.5 \mathrm{ps}$ on the KP-VLBA/OV-VLBA baseline, up to $16.1 \mathrm{ps}$ on the BR-VLBA/MK-VLBA baseline. The wrms over all baselines is only $6.0 \mathrm{ps}$, equivalent to $1.8 \mathrm{~mm}$. By comparison, the average delay formal errors are $7.7 \mathrm{ps}$ on KP-VLBA/OV-VLBA and $17.3 \mathrm{ps}$ on BR-VLBA/MK-VLBA. In a similar comparison between the Mark 3 and Mark 4 correlators and post-processing software at Bonn University, Nothnagel et al. (2002) found an average wrms difference of 21.1 ps on 6 long intercontinental baselines.

\section{Geodetic analysis}

In our analysis we used all available VLBI observations from August 03, 1979 through October 04, 2007, including 132 observing sessions with the VLBA. The differences between the observed ionosphere-free linear combinations of dual-frequency group delays and theoretical group delays are used in the right hand side of the observation equations in the least squares parameter estimation procedure.

Computation of theoretical time delays in general follows the approach outlined in the IERS Conventions (McCarthy and Petit, Eds 2004) and presented in detail by Sovers et al. (1998) with some minor refinements. The most significant ones are the follow- 
Table 4 A comparison of X-band group delays and phase delay rates between a subset of the rdv22 session processed through the Haystack Mark 4 correlator/Fourfit processing system versus the VLBA Correlator/AIPS processing system.

\begin{tabular}{lrrrr}
\hline & & & & \\
WRMS Differences \\
Baseline & \#pts & $\begin{array}{r}\text { Length } \\
(\mathrm{km})\end{array}$ & $\begin{array}{r}\text { Delays } \\
(\mathrm{ps})\end{array}$ & $\begin{array}{r}\text { Rates } \\
10^{-15}\end{array}$ \\
\hline LA-VLBA /PIETOWN & 135 & 237 & 5.1 & 31.3 \\
KP-VLBA /PIETOWN & 124 & 417 & 3.6 & 14.1 \\
KOKEE /MK-VLBA & 169 & 508 & 5.1 & 35.1 \\
KP-VLBA /LA-VLBA & 125 & 652 & 5.2 & 15.8 \\
KP-VLBA /OV-VLBA & 171 & 845 & 2.5 & 61.1 \\
OV-VLBA /PIETOWN & 97 & 973 & 7.4 & 30.0 \\
LA-VLBA /OV-VLBA & 76 & 1088 & 5.6 & 22.1 \\
BR-VLBA /OV-VLBA & 176 & 1215 & 3.3 & 30.4 \\
BR-VLBA /LA-VLBA & 104 & 1757 & 3.0 & 30.3 \\
BR-VLBA /PIETOWN & 170 & 1806 & 7.8 & 60.4 \\
BR-VLBA /KP-VLBA & 183 & 1914 & 6.8 & 20.8 \\
BR-VLBA /GILCREEK & 143 & 2482 & 4.0 & 10.2 \\
GILCREEK/OV-VLBA & 24 & 3584 & 6.1 & 51.9 \\
MK-VLBA /OV-VLBA & 124 & 4015 & 7.4 & 7.4 \\
KOKEE /OV-VLBA & 59 & 4220 & 8.4 & 36.8 \\
GILCREEK/PIETOWN & 103 & 4225 & 4.8 & 43.3 \\
GILCREEK/KP-VLBA & 98 & 4322 & 3.8 & 9.4 \\
BR-VLBA /MK-VLBA & 162 & 4399 & 16.1 & 23.2 \\
KP-VLBA /MK-VLBA & 43 & 4467 & 13.3 & 33.2 \\
BR-VLBA /KOKEE & 112 & 4469 & 9.2 & 11.3 \\
GILCREEK/KOKEE & 95 & 4728 & 15.4 & 36.5 \\
KOKEE / KP-VLBA & 113 & 4736 & 5.6 & 24.9 \\
MK-VLBA / PIETOWN & 38 & 4796 & 9.0 & 39.7 \\
GILCREEK/MK-VLBA & 21 & 4923 & 11.6 & 53.8 \\
LA-VLBA /MK-VLBA & 89 & 4970 & 9.3 & 51.1 \\
KOKEE /PIETOWN & 105 & 5040 & 7.9 & 77.2 \\
ALL & & & & \\
\hline & 2859 & - & 6.0 & 36.1 \\
\hline
\end{tabular}

ing. The expression for time delay derived by Kopeikin and Schäfer (1999) in the framework of general relativity was used. The displacements caused by the Earth's tides were computed using a rigorous algorithm (Petrov and Ma 2003) with a truncation at a level of $0.05 \mathrm{~mm}$ using the numerical values of the generalized Love numbers presented by Mathews (2001). The displacements caused by ocean loading were computed by convolving the Greens' functions with ocean tide models using the NLOADF algorithm of Agnew (1997). The GOT00 model (Ray 1999) of diurnal and semi-diurnal ocean tides, the NAO99 model (Matsumoto et al. 2000) of ocean zonal tides, the equilibrium model (Petrov and Ma 2003) of the pole tide, and the tide with period of 18.6 years were used. Atmospheric pressure loading was computed by convolving the Greens' functions with the output of the atmosphere NCEP Reanalysis numerical model (Kalnay et al. 1996). The algorithm of computations is described in full details in Petrov and Boy (2004). The empirical model of harmonic variations in the Earth orientation parameters heo_20070802 derived from VLBI observations according to the method proposed by Petrov (2007) was used. The time series of
UT1 and polar motion derived by the Goddard operational VLBI solutions were used as a priori. Displacement of the VLBI reference points due to antenna thermal expansion was not modeled.

The ionosphere contribution to group delay is considered to be reciprocal to the square of frequency. Therefore, there exists the linear combination of Xband and S-band delays that is ionosphere-free. No additional ionosphere model was applied. The contribution of higher terms to the ionosphere delay as was shown by Hawarey et al. (2005) is less than 9 ps. Its maximum contribution to estimates of site positions is below $0.5 \mathrm{~mm}$ and it was ignored.

The a priori path delay in the atmosphere caused by the hydrostatic component was calculated as a product of the zenith path delay computed on the basis of surface pressure using the Saastamoinen (1972a,b) expression with corrections introduced by Davis et al. (1985) and the so-called hydrostatic mapping function (Niell 1996). The mapping function describes the dependence of path delay on the angle between the local axis of symmetry of the atmosphere and the direction to the observed sources.

Several solutions were produced. Each solution used the basic parameterization which was common for all runs and a specific parameterization for an individual solution. Basic parameters belong to one of the three groups:

- global (over the entire data set): positions of 3089 sources.

- local (over each session): tilts of the local symmetric axis of the atmosphere (also known as "atmospheric azimuthal gradients") for all stations and their rates, station-dependent clock functions modeled by second order polynomials, baseline-dependent clock offsets, daily nutation offset angles.

- segmented (over 20-60 minutes): coefficients of linear spline that model atmospheric path delay (20 minutes segment) and clock function (60 minutes segment) for each station. The estimates of clock function absorb uncalibrated instrumental delays in the data acquisition system.

The rate of change for the atmospheric path delay and clock function between adjacent segments was constrained to zero with weights reciprocal to $1.1 \cdot 10^{-14}$ and $2 \cdot 10^{-14}$, respectively, in order to stabilize solutions. The weights of observables were computed as $w=1 / \sqrt{\sigma_{o}^{2}+r^{2}(b)}$, where $\sigma_{o}$ is the formal uncertainty of group delay estimation and $r(b)$ is the baselinedependent reweighting parameter that was evaluated in a trial solution to make the ratio of the weighted sum of the squares of residuals to its mathematical expectation to be close to unity. 


\subsection{Baseline analysis}

In the preliminary stage of data analysis, in addition to basic parameters we estimated the length of each baseline at each session individually. The purpose of this solution was to determine possible non-linearity in station motion, to detect possible outliers, and to evaluate statistics related to systematic errors. The baseline length is invariant with respect to a linear coordinate transformation that affects all the stations of the network. Therefore, changes in baseline lengths are related to either physical motion of one station with respect to another or to systematic errors specific to observations at the stations of the baseline.

We present in Figures 4.1-4.1 examples of length evolutions for a very stable intra-plate baseline and for a rapidly stretching inter-plate baseline.

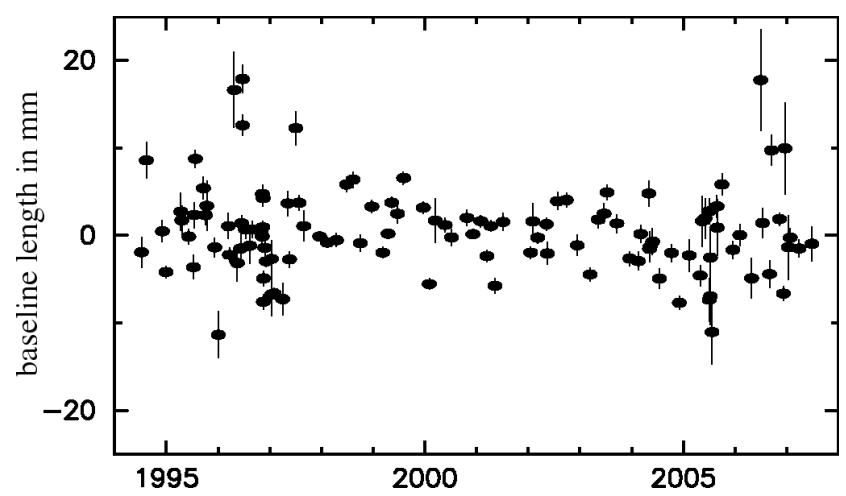

Fig. 3 Residual lengths of the intra-plate baseline HN-VLBA/FDVLBA with respect to the average value of $3623021.2526 \mathrm{~m}$. The wrms $3.7 \mathrm{~mm}$.



Fig. 4 Residual lengths of the inter-plate baseline MK-VLBA/SCVLBA with respect to the average value of $8611584.6972 \mathrm{~m}$. The wrms $9.2 \mathrm{~mm}$

As we see, the tectonic motion has shifted station MK-VLBA, located on the fast Pacific plate, by more than 0.5 meters over the existence of the array.

No significant outliers, and no jumps exceeding $2 \mathrm{~cm}$ were found in examining plots of the baseline length evolution. Significant non-linear motion was found only on baselines with station PIETOWN (Figure 5). Analysis of GPS data from the permanent IGS station PIE1 located within $61.8 \mathrm{~m}$ of the VLBI station (Figure 2) does not show a similar pattern.

\subsection{Global analysis}

The purpose of the global solution is to determine the best model of station motion. In general, the model of motion of the $k$ th station can be represented in this form:

$$
\begin{gathered}
\mathbf{r}_{k}=\mathbf{r}_{o k}+\dot{\mathbf{r}}_{k} t+\sum_{j=1-m}^{n_{k}} \mathbf{f}_{k j} B_{j}^{m}\left(t ; t_{1-m, k}, \ldots t_{n_{k}, k}\right)+ \\
\sum_{i}^{n_{h}}\left(\mathbf{h}_{k i}^{c} \cos \left(\alpha_{i}+\omega_{i} t\right)+\mathbf{h}_{k i}^{s} \sin \left(\alpha_{i}+\omega_{i} t\right)\right)
\end{gathered}
$$

Here $\mathbf{r}_{o k}$ is the position of the $k$ th station at the reference epoch when $\mathrm{t}=0, \dot{\mathbf{r}}_{k}$ is the linear station velocity, $B_{j}^{m}\left(t ; t_{1-m}, \ldots, t_{n_{k}, k}\right)$ is the B-spline of $m$ th degree defined on a knot sequence $t_{1-m, k}, \ldots, t_{n_{k}, k}$ that is unique for each station and not necessarily equidistant with the $j$ th pivotal element. Properties of B-spline function are discussed in full details in de Boor (1978); Nürnberger (1989). The first two terms in 1 describe the linear station motion, the last one describes harmonic motion, and the third term describes the non-linear, anharmonic motion with possible discontinuities caused by seismic events or antenna repair.

The parameters of the non-linear model of motion for selected sites, the frequencies of harmonic site position variations, the degree of the B-splines, and the sequences of knots on which the B-splines are defined, were selected manually. Several trial solutions were made, and the series of the baseline length estimates were scrutinized. The parameters of the non-linear model were adjusted until the plots of residuals showed no systematics. The stations for estimation of harmonic position variations were selected on the basis of their observational history. Only those stations that participated in observations at least once every three months for at least three years were selected to avoid strong correlation between estimates of harmonic site position variations and other parameters.

We estimated non-linear anharmonic motion at 18 stations, including two VLBA stations PIETOWN and MK-VLBA. The degree of B-spline was 0 for MK-VLBA and 2 for PIETOWN. The epochs of B-spline knots are presented in Table 5. 
Table 5 Epochs of knots of B-spline for modeling a non-linear anharmonic motion of two VLBA stations.

\begin{tabular}{llll}
\hline PIETOWN & 1988.09 .08 & MK-VLBA & 1993.07 .19 \\
PIETOWN & 1993.03 .01 & MK-VLBA & 2000.04 .02 \\
PIETOWN & 1996.01 .01 & MK-VLBA & 2006.10 .15 \\
PIETOWN & 1998.01 .01 & MK-VLBA & 2007.08 .10 \\
PIETOWN & 2000.01 .01 & & \\
PIETOWN & 2002.01 .01 & & \\
PIETOWN & 2004.01 .01 & & \\
PIETOWN & 2007.08 .10 & & \\
\hline
\end{tabular}

We ran a special global solution ${ }^{4}$, where in addition to parameters estimated in the previous solution, we estimated as global parameters quantities $\mathbf{r}_{o k}, \dot{\mathbf{r}}_{k}$ for all stations, quantities $\mathbf{h}_{k i}^{c}, \mathbf{h}_{k i}^{s}$ for all VLBA stations and 25 selected sites, and quantities $\mathbf{f}_{k j}$ for five stations. The polar motion, UT1, and their first time derivatives were also estimated.

\subsection{Required minimum constraints}

Equations of light propagation are differential equations of the second order. Their solution does not allow determining specific coordinates of sources and stations, but rather a family of coordinate sets. Boundary conditions should be formulated either implicitly or explicitly in the form of constraints in order to select an element from these sets. These boundary conditions cannot in principle be determined from the observations. Thus, observations alone are not sufficient to evaluate station positions and source coordinates. Coordinates are determined from observations in the form of observation equations and boundary conditions in the form of constraint equations.

Expressions for VLBI path delays are invariant with respect to a group of coordinate transformation that involves translation and rotation of site positions at a reference epoch, their first time derivatives, and rotation of source coordinates. In order to remove the rank deficiency, we imposed constraints in the form

$$
\begin{array}{ll}
\sum_{k}^{n_{s}}\left(\Delta \mathbf{r}_{o k} \times \mathbf{r}_{o k}\right) /\left|\mathbf{r}_{o k}\right|=\mathrm{const} & \sum_{k}^{n_{s}} \Delta \mathbf{r}_{o k}=\mathrm{const} \\
\sum_{k}^{n_{s}}\left(\Delta \dot{\mathbf{r}}_{k} \times \mathbf{r}_{o k}\right) /\left|\mathbf{r}_{o k}\right|=\mathrm{const} \quad \sum_{k}^{n_{s}} \Delta \dot{\mathbf{r}}_{k}=\mathrm{const} \\
\sum_{a}^{q} \Delta \mathbf{s}_{a} \times \mathbf{s}_{a}=\mathrm{const},
\end{array}
$$

where $\mathbf{s}_{a}$ is the coordinate vector of $a$ th source, $n_{s}$ is the number of stations that participate in constraints, and $q$ is the number of sources that participate in constraints.

4 Listing of this solution is available at http://astrogeo.org/vlbi/solutions/2007d_adv
The pairs of parameters $\mathbf{r}_{o k}, \mathbf{f}_{k i}$ and $\dot{\mathbf{r}}_{k}, \mathbf{f}_{k i}$ are linearly dependent, and pairs of parameters $\mathbf{f}_{k i}, \mathbf{h}_{k i}^{c}$, and $\mathbf{f}_{k i}, \mathbf{h}_{k i}^{s}$ may be highly correlated depending on frequencies. In order to avoid rank deficiency of a system of observation equations, the following decorrelation constraints are to be imposed for each frequency of the harmonic constituents:

$$
\begin{aligned}
& \sum_{j=1-m}^{m-1} f_{j} \int_{-\infty}^{+\infty} B_{j}^{m}(t) \cos \omega_{i} t d t=\text { const } \\
& \sum_{j=1-m}^{m-1} f_{j} \int_{-\infty}^{+\infty} B_{j}^{m}(t) \sin \omega_{i} t d t=\text { const. }
\end{aligned}
$$

Decorrelation constraints between the estimates of B-spline coefficients, the estimate of mean site position $\mathbf{r}_{o k}$ and linear velocity $\dot{\mathbf{r}}_{k}$ (in the case if the degree of B-spline $m>0$ ) are to be imposed as well:

$$
\begin{aligned}
& \sum_{j=1-m}^{m-1} f_{j} \int_{-\infty}^{+\infty} B_{j}^{m}(t) d t=\text { const } \\
& \sum_{j=1-m}^{m-1} f_{j} \int_{-\infty}^{+\infty} t B_{j}^{m}(t) d t=\text { const. }
\end{aligned}
$$

The integrals $3-4$ can be evaluated analytically (Nürnberger 1989).

Similar to coordinates, the adjustments of harmonic variations in coordinates are invariant with respect to a group of transformations that involve translation and rotation. In order to remove the rank deficiency associated with this group of transformations, we imposed the following constraints:

$$
\begin{array}{rlrl}
\sum_{k}^{n_{s}}\left(\mathbf{h}_{k i}^{c} \times \mathbf{r}_{k}\right) /\left|\mathbf{r}_{k}\right| & =\text { const } & \sum_{k}^{n_{h}} \mathbf{h}_{k i}^{c}=\mathrm{const} \\
\sum_{k}^{n_{s}}\left(\mathbf{h}_{k i}^{s} \times \mathbf{r}_{k}\right) /\left|\mathbf{r}_{k}\right|=\mathrm{const} & \sum_{k}^{n_{h}} \mathbf{h}_{k i}^{s}=\mathrm{const} .
\end{array}
$$

In our solutions, we set the constants in equations $2-$ 5 to zero. 4.4 Motion of the reference point due to antenna
instability

The residuals of time series of baseline length estimates with station PIETOWN with respect to a linear fit show a significant systematic behavior. In our efforts to understand the origin of this behavior, we investigated the effect of variations of up to $4^{\prime}$ in the antenna's tilt, made evident from pointing measurements. 


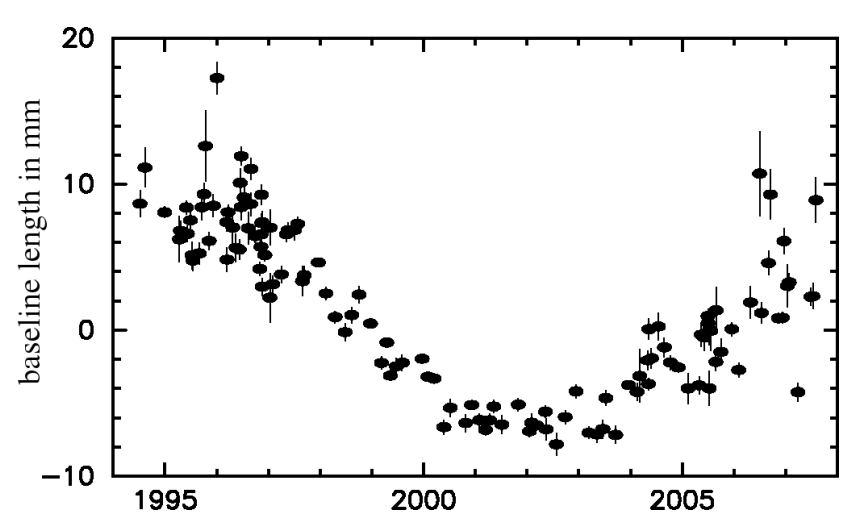

Fig. 5 Length of the inter-plate baseline KP-VLBA/PIETOWN with respect to the average value of $417009.1252 \mathrm{~m}$. The baseline length estimates exhibit a non-linear motion caused by variations in the antenna's tilt.

When an antenna is pointed at a source, the actual azimuth and elevation commands sent to the antenna control unit are the expected azimuth and elevation on the sky, including refraction, plus offsets that adjust for imperfections in the antenna and encoders. The offsets typically amount to a few arc-minutes. They may be calculated using pointing equation 6 in which the imperfections are parameterized in terms of expected physical effects. The same equation, with site specific values for the coefficients, is used at all VLBA stations. The coefficients for the different effects in equation 6 are determined in pointing observations designed for that purpose.

$$
\begin{array}{rlrl}
\Delta \mathrm{Az}= & T_{e} \sin \mathrm{Az} & & +T_{n} \cos \mathrm{Az} \\
& +a_{0} & & +a_{1} \cos \mathrm{El} \\
& +a_{2} \sin \mathrm{El} & & +a_{3} \cos 2 \mathrm{Az} \\
& +a_{4} \sin 2 \mathrm{Az} & & +H_{a}(\mathrm{Az}, \mathrm{El}) \\
\Delta \mathrm{El}= & -T_{e} \cos \mathrm{Az} \sin \mathrm{El} & & +T_{n} \sin \mathrm{Az} \sin \mathrm{El} \\
& +e_{0} & & +e_{1} \cos \mathrm{El} \\
& +e_{2} \cos \mathrm{El} & & +e_{3} \cos \mathrm{El} \sin \mathrm{Az} \\
& +e_{4} \cos \mathrm{El} \cos \mathrm{Az} & & +e_{5} \cos \mathrm{El} \sin 2 \mathrm{Az} \\
& +e_{6} \cos \mathrm{El} \cos 2 \mathrm{Az} & +H_{e}(\mathrm{Az}) .
\end{array}
$$

$H_{a}(\mathrm{Az}, \mathrm{El})$ and $H_{e}(\mathrm{Az})$ are the contribution to azimuth and elevation offsets due to rail height variations. The rail height $\mathrm{H}(\mathrm{Az})$ was determined for the VLBA antennas by leveling for every $3^{\circ}$ along the circular rail track of $15.24 \mathrm{~m}$ diameter. A three-parameter model $H_{o}+H_{c} \cos \mathrm{Az}+\mathrm{H}_{\mathrm{s}} \sin \mathrm{Az}$ was fit to these raw measurements and subtracted. The effect of rail height variations is complicated by the fact that there are 4 wheels, each responding to the rail height at its location and distorting the antenna mount accordingly. A simple model, based on analyses by B. Clark and J. Thunborg (private communication), was developed to describe the effect of rail height:

$$
\begin{aligned}
& H_{a}(\mathrm{Az}, \mathrm{El})= \\
& h_{a 1} \sin \mathrm{El}\left[\mathrm{H}\left(\mathrm{Az}+45^{\circ}\right)-\mathrm{H}\left(\mathrm{Az}-45^{\circ}\right)\right] \\
& +h_{a 2} \sin \mathrm{El}\left[\mathrm{H}\left(\mathrm{Az}+135^{\circ}\right)-\mathrm{H}\left(\mathrm{Az}-135^{\circ}\right)\right] \\
& -h_{a 3} \cos \mathrm{El}\left[\mathrm{H}\left(\mathrm{Az}+45^{\circ}\right)-\mathrm{H}\left(\mathrm{Az}-45^{\circ}\right)\right] \\
& +h_{a 4} \cos \mathrm{El}\left[\mathrm{H}\left(\mathrm{Az}+135^{\circ}\right)-\mathrm{H}\left(\mathrm{Az}-135^{\circ}\right)\right] \\
& \quad=\quad \\
& \quad h_{e 1} H\left(\mathrm{Az}+135^{\circ}\right) \\
& +h_{e 2} H\left(\mathrm{Az}-135^{\circ}\right) \\
& -h_{e 3} H\left(\mathrm{Az}+45^{\circ}\right) \\
& -h_{e 4} H\left(\mathrm{Az}-45^{\circ}\right)
\end{aligned}
$$

It should be noted that the eight coefficients $h_{a 1}$ $h_{a 4}$ and $h_{e 1}-h_{e 4}$ are linearly dependent. The equations 7 can be reduced to linear combinations of two independent parameters. Since all VLBA antennas have identical design, these parameters are considered to be the same for all antennas. They were determined for several antennas with the largest rail height variations and then kept fixed. The method is described in details by Walker (1999).

The parameters of pointing equations $6, T_{e}, T_{n}, a_{0^{-}}$ $a_{4}, e_{0}-e_{6}$, are determined using least squares fits to measurements of residual pointing offsets. These measurements are made at 13 observing bands right after the weekly maintenance day, during "startup" observations designed to help verify proper operation of the telescope. Special targeted pointing observations, often of order 10 hours in length, are made during other times that the antennas are not needed for interferometer observations. These special observations often concentrate on the $22 \mathrm{GHz}$ and $43 \mathrm{GHz}$ bands.

Such measurements are made by recording the total power output in baseband channels of $16 \mathrm{MHz}$ bandwidth attached to both left and right circular polarization output from the receiver while pointing at each of 10 positions near the expected position of a strong source. The 10 points are off-source, half-power, onsource, half-power, and off-source, in both azimuth and elevation, with the two half-power and off positions being on opposite sides of the source. The off positions are about 6 beam half-widths from the source, but, for the elevation pattern, much of that offset is in azimuth to allow even steps in elevation. The full width of half maximum of the beam at $22 \mathrm{GHz}$ is $1^{\prime} .9$. The residual pointing offset and gain are determined by subtracting interpolated off-source powers from the on-source and half-power number, and then fitting for a peak amplitude and position. The even steps in elevation of the elevation scan make the removal of gradients in eleva- 
a) East component

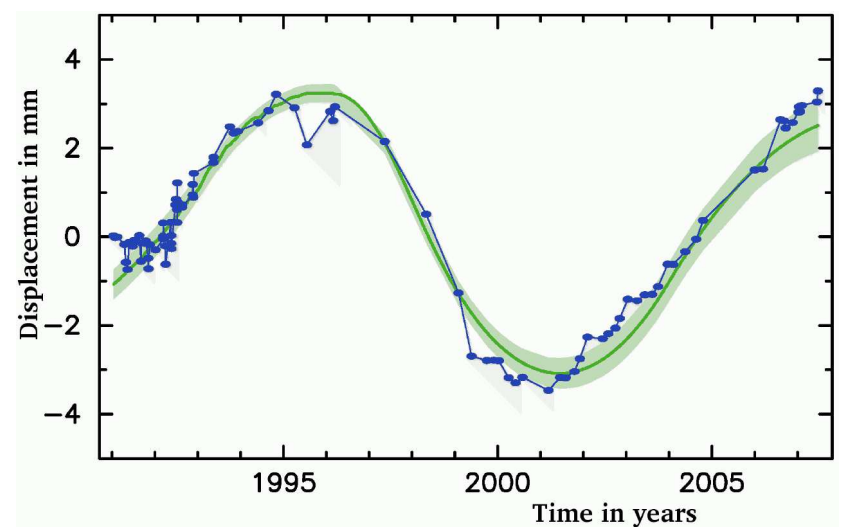

b) North component

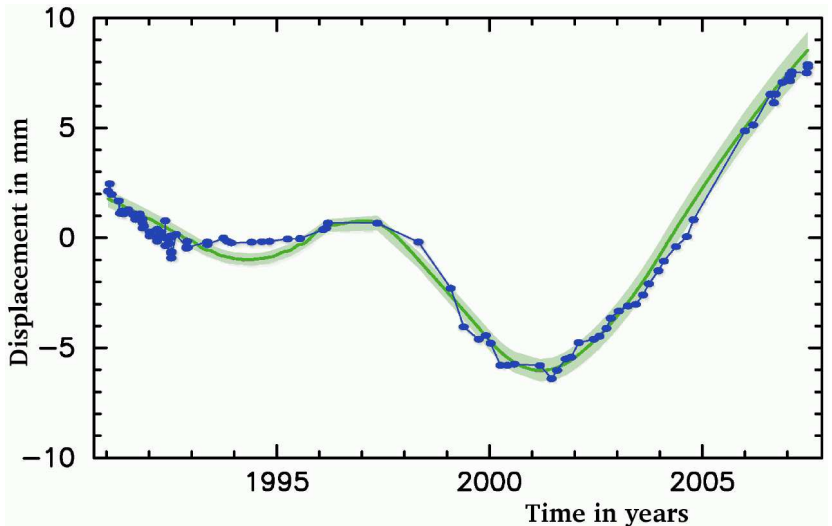

Fig. 6 Nonlinear motion of station PIETOWN as estimated from global VLBI analysis (smooth line) and changes in the tilt converted to displacement of the antenna reference point, considering rigid rotation of the antenna. The shadow shows the 1- $\sigma$ formal uncertainties of the displacement estimate.

a) East component

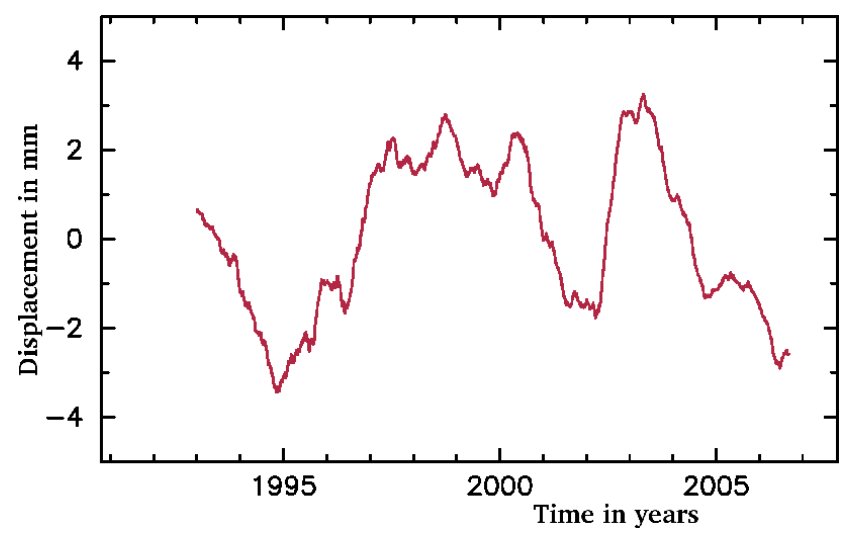

b) North component

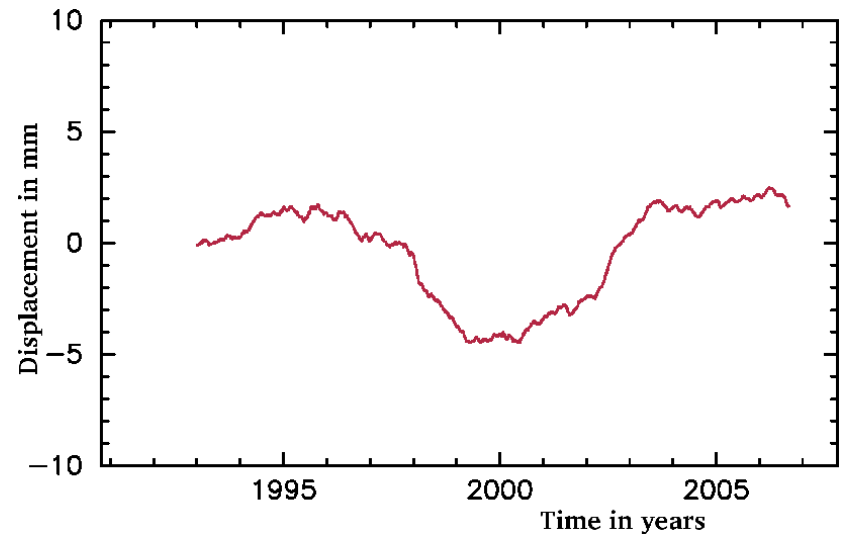

Fig. 7 Nonlinear motion of station PIE1 as estimated from GPS time series after subtracting the mean position, linear velocity, annual and semi-annual position variations from the LSQ fit of daily estimates of station positions generated by Steigenberger et al. (2006) and subsequent smoothing.

tion, such as naturally arise from the atmosphere, more effective.

Throughout this process, a modulated noise calibration signal of known equivalent temperature is injected in front of the receiver amplifiers and synchronously detected along with the total power. This allows calibration of the total power and the power contributed by the target source in terms of antenna temperature. Using sources of known flux density the gain is determined as a ratio of the source flux density to the antenna temperature. Continuum sources used for pointing observations must be stronger than 5 Jy to provide enough power so that they are not masked by normal atmospheric fluctuations.

Occasionally, all pointing observations made over the course of about three months are gathered together for a single fit. Such an analysis typically involves about 1000 separate measurements at each of the $22 \mathrm{GHz}$ and
$43 \mathrm{GHz}$ bands. These high frequency bands are used to determine most of the pointing equation parameters because, with their smaller beam widths, they produce more accurate pointing measurements.

Prior to a fit, the effects of beam squint are removed. This is the offset between the left and right circular polarized beams caused by the asymmetric geometry of the VLBA antennas. The offset amounts to about 5\% of the beam Full Width Half Maximum (FWHM). An antenna is commanded to point to a position half way between the right and left circular polarization beams regardless of the polarization being observed, so it is always pointed about $2.5 \%$ of the FWHM away from the beam center.

In the fit for the pointing parameters, some of the terms described above are held fixed. The axis nonperpendicularity $a_{2}$ is generally held to zero. 
Some of the terms of the pointing equation are highly correlated, so the allocation of offsets to individual physical effects may have large errors. But as long as a set of terms determined in a single solution are used, the derived pointing offsets will be good. An example is the constant, $\sin (\mathrm{El})$, and $\cos (\mathrm{El})$ terms in the elevation equation (Equation 7 for $\Delta E l$ ) that have to be determined from data that span only about $75^{\circ}$ in elevation. The sum of the effects of these terms is well determined and that is all that matters for pointing.

The terms $T_{e}$ and $T_{n}$ give the east and north tilts of the fixed azimuthal axis. They depend on $\sin (\mathrm{Az})$ and $\cos (\mathrm{Az})$. Since the measurements cover all azimuths, these estimates are not significantly correlated with other terms. A classic example of tilt is the leaning tower in Pisa. The tilt can be considered as a small rotation of the antenna as a whole with respect to a certain point. This rotation shifts the position of the reference point.

From our global solution we derived an empirical model of the PIETOWN reference point position variations by estimating the B-spline coefficients. From these coefficients we computed an empirical time series of PIETOWN displacements. The empirical time series of horizontal displacements were fit using time series of the measured north and east tilts derived from antenna pointing, and a single admittance factor was adjusted. The estimate of the adjusted parameter is $20.5 \pm 0.2 \mathrm{~m}$. Its physical meaning is the distance between the center of rotation that causes the tilt and the displacement of the antenna reference point. As Figure 4.4 shows, the empirical model agrees with the tilt measurements within its formal uncertainties, at a $0.5 \mathrm{~mm}$ level. Thus, the anomalous PIETOWN horizontal non-linear motion can be explained almost entirely by variations in the tilt of the PIETOWn antenna. The site position series (Steigenberger et al. 2006) from the nearby GPS station PIE1, located at a distance of $61.8 \mathrm{~m}$ from the VLBI antenna, shows some similarity in non-linear motion in the north component (Figure 4.4, correlation coefficient 0.87 ), but not in the east component (correlation coefficient -0.73). The origin of the non-linear motion of PIETOWN has not been firmly established, but is thought to be settling of the ground beneath the telescope. The antenna is on sloped ground and is leaning into the slope.

\subsection{Analysis of the VLBA array velocity field}

To address the question of stability of the VLBA array, we would like to determine if any part of the array exhibits only rigid horizontal motion, i.e. with relative horizontal velocities close to zero. Since station velocity estimates depend not only on observations but on constraint equations with an arbitrary right hand side, the estimates of motion of the VLBA array as a whole is also subject to an arbitrary translation and rotation. This means that all velocity vectors of the network stations can be transformed as

$\mathbf{v}_{n k}=\mathbf{v}_{o k}+\widehat{\mathcal{M}}_{k} \mathbf{s}$,

where $\widehat{\mathcal{M}}_{k}$ is the transformation matrix for the $k$ th station, and $\mathbf{s}$ is an 6-vector of small arbitrary translationrotation:

$$
\begin{aligned}
\widehat{\mathcal{M}}_{k} & =\left(\begin{array}{rrrrrr}
1 & 0 & 0 & 0 & r_{3 k} & -r_{2 k} \\
0 & 1 & 0 & -r_{3 k} & 0 & r_{1 k} \\
0 & 0 & 1 & r_{2 k} & -r_{1 k} & 0
\end{array}\right) \\
\mathbf{s} & =\left(\begin{array}{rrrrrr}
T_{1} & T_{2} & T_{3} & \Omega_{1} & \Omega_{2} & \Omega_{3}
\end{array}\right)^{\top} .
\end{aligned}
$$

We can find such a vector $\mathbf{s}$ that the transformed velocity field will have some desirable properties. This is equivalent to running a new solution with different right hand sides in constraint equations $2-5$.

Equation 8 is transformed to a local topocentric coordinate system of the $k$ th station by multiplying it by the projection matrix $\widehat{\mathcal{P}}_{k}$ :

$\widehat{\mathcal{P}}_{k} \widehat{\mathcal{M}}_{k} \mathbf{s}=\mathbf{u}_{k}-\widehat{\mathcal{P}}_{k} \mathbf{v}_{o k}$,

where $\mathbf{u}$ is the vector of the station velocity in a topocentric coordinate system after the transformation. We can find vector $\mathbf{s}$ from equation 10 if we define $\mathbf{u}_{k}$ according to the model of rigid motion. We split the set of stations into two subsets. The first set called "defining", exhibits only rigid motion, either horizontal or vertical, or both. Stations from the second set, called "free", have non-negligible velocities with respect to the rigid motion.

We extended our analysis to four non-VLBA stations located in the vicinity of VLBA antennas in order to investigate the continuity of the velocity field. The set of defining stations was found by an extensive trial. The residual velocity of defining stations with respect to the rigid motion was examined. We found a set of 7 defining stations for which the residual velocity does not exceed $3 \sigma$ (Table 6 ). Six stations qualify as horizontal defining stations and three as vertical defining stations.

Using equation 10 for the horizontal components of the 6 horizontal defining stations, we set the horizontal components of vector $\mathbf{u}_{k}$ to zero, and build a system of linear equations. This system is augmented by adding equations for the vertical components of the 3 vertical defining stations and we also set the vertical components of vector $\mathbf{u}_{k}$ to zero. When the number 
Table 6 Station local topocentric velocities with respect to the rigid North American plate. Units: mm/yr. The quoted uncertainties are re-scaled 1- $\sigma$ standard errors. The last column indicates whether the station was used as defining for horizontal (h) or vertical (v) motion of the plate.

\begin{tabular}{|l|r|r|r|l|}
\hline Station & \multicolumn{1}{|c|}{ Up } & \multicolumn{1}{|c|}{ East } & \multicolumn{1}{|c|}{ North } & Def \\
\hline BR-VLBA & $0.0 \pm 0.1$ & $1.9 \pm 0.3$ & $-0.3 \pm 0.4$ & $\mathrm{~V}$ \\
FD-VLBA & $1.2 \pm 1.0$ & $0.4 \pm 0.2$ & $-0.1 \pm 0.2$ & $\mathrm{~h}$ \\
HN-VLBA & $0.2 \pm 0.3$ & $-0.1 \pm 0.2$ & $-0.1 \pm 0.2$ & $\mathrm{hv}$ \\
KP-VLBA & $2.3 \pm 1.0$ & $-0.2 \pm 0.2$ & $0.1 \pm 0.2$ & $\mathrm{~h}$ \\
LA-VLBA & $1.3 \pm 0.8$ & $0.0 \pm 0.3$ & $0.0 \pm 0.2$ & $\mathrm{~h}$ \\
MK-VLBA & $0.9 \pm 1.1$ & $-55.0 \pm 1.4$ & $52.5 \pm 1.1$ & \\
NL-VLBA & $-1.1 \pm 0.5$ & $0.0 \pm 0.2$ & $0.1 \pm 0.3$ & $\mathrm{~h}$ \\
OV-VLBA & $2.0 \pm 0.8$ & $-6.0 \pm 0.3$ & $5.0 \pm 0.4$ & \\
PIETOWN & $2.1 \pm 0.9$ & $-0.4 \pm 0.3$ & $-1.5 \pm 0.3$ & \\
SC-VLBA & $-1.2 \pm 1.2$ & $19.0 \pm 0.8$ & $5.3 \pm 0.4$ & \\
GILCREEK & $3.0 \pm 1.4$ & $4.0 \pm 0.6$ & $-10.2 \pm 0.7$ & \\
GGAO7108 & $-0.9 \pm 0.5$ & $-0.4 \pm 0.3$ & $-0.5 \pm 0.3$ & \\
KOKEE & $2.7 \pm 1.0$ & $-54.3 \pm 1.3$ & $52.9 \pm 1.2$ & \\
WESTFORD & $-0.3 \pm 0.3$ & $-0.1 \pm 0.2$ & $0.0 \pm 0.2$ & $\mathrm{hv}$ \\
\hline
\end{tabular}

of equations exceeds 6 , the system becomes redundant. We solve it by LSQ with a full weight matrix $\widehat{\mathcal{W}}$ :

$\widehat{\mathcal{W}}=\left(\widehat{\mathcal{P}}_{a} \operatorname{Cov}\left(\mathbf{v}_{o}, \mathbf{v}_{o}^{\top}\right) \widehat{\mathcal{P}}_{a}^{\top}+\widehat{\mathcal{A}}\right)^{-1}$,

where $\widehat{\mathcal{P}}_{a}$ is a block-diagonal matrix formed from matrices $\widehat{\mathcal{P}}_{k}, \widehat{\mathcal{A}}$ is a diagonal reweighting matrix with an additive correction to weights. Values of $(0.4 \mathrm{~mm} / \text { year })^{2}$ for both horizontal and vertical velocity components, corresponding to a conservative measure of errors, were used in the matrix $\widehat{\mathcal{A}}$ in our solution.

Then, transformation 10 and the rotation to the local topocentric coordinate system were applied to both defining and free stations. The covariance matrix for velocity estimates of free stations was computed as:

$$
\begin{aligned}
\operatorname{Cov}\left(\mathbf{v}_{n}, \mathbf{v}_{n}^{\top}\right)= & \widehat{\mathcal{P}}_{a} \operatorname{Cov}\left(\mathbf{v}_{o}, \mathbf{v}_{o}^{\top}\right) \widehat{\mathcal{P}}^{\top}{ }_{a} \\
& +\widehat{\mathcal{P}}_{a} \widehat{\mathcal{M}} \operatorname{Cov}\left(\mathbf{s}, \mathbf{s}^{\top}\right) \widehat{\mathcal{M}}^{\top} \widehat{\mathcal{P}}^{\top}{ }_{a}
\end{aligned}
$$

and for defining stations as

$$
\begin{aligned}
\operatorname{Cov}\left(\mathbf{v}_{n}, \mathbf{v}_{n}^{\top}\right)= & \widehat{\mathcal{P}}_{a} \operatorname{Cov}\left(\mathbf{v}_{o}, \mathbf{v}_{o}^{\top}\right) \widehat{\mathcal{P}}^{\top}{ }_{a} \\
& +\widehat{\mathcal{P}}_{a} \widehat{\mathcal{M}} \operatorname{Cov}\left(\mathbf{s}, \mathbf{s}^{\top}\right) \widehat{\mathcal{M}}^{\top} \widehat{\mathcal{P}}^{\top}{ }_{a} \\
& +\widehat{\mathcal{P}}_{a} \operatorname{Cov}\left(\mathbf{s}, \mathbf{s}^{\top}\right) \widehat{\mathcal{M}} \widehat{\mathcal{W}} \operatorname{Cov}\left(\mathbf{v}_{o}, \mathbf{v}_{o}^{\top}\right) \widehat{\mathcal{P}}^{\top}{ }_{a}(13) \\
& +\widehat{\mathcal{P}}_{a} \operatorname{Cov}\left(\mathbf{v}_{o}, \mathbf{v}_{o}^{\top}\right) \widehat{\mathcal{W}} \widehat{\mathcal{M}}^{\top} \operatorname{Cov}\left(\mathbf{s}, \mathbf{s}^{\top}\right) \widehat{\mathcal{P}}^{\top}{ }_{a}
\end{aligned}
$$

The latter expression takes into account statistical dependence of the a priori velocity $\mathbf{v}_{o}$ and the vector $\mathbf{s}$.

The results are presented in Table 6 . It is remarkable that there exists a set of 6 stations spread over distances of 1-3 thousand kilometers with an average residual horizontal velocity of only $0.2 \mathrm{~mm} / \mathrm{yr}$.

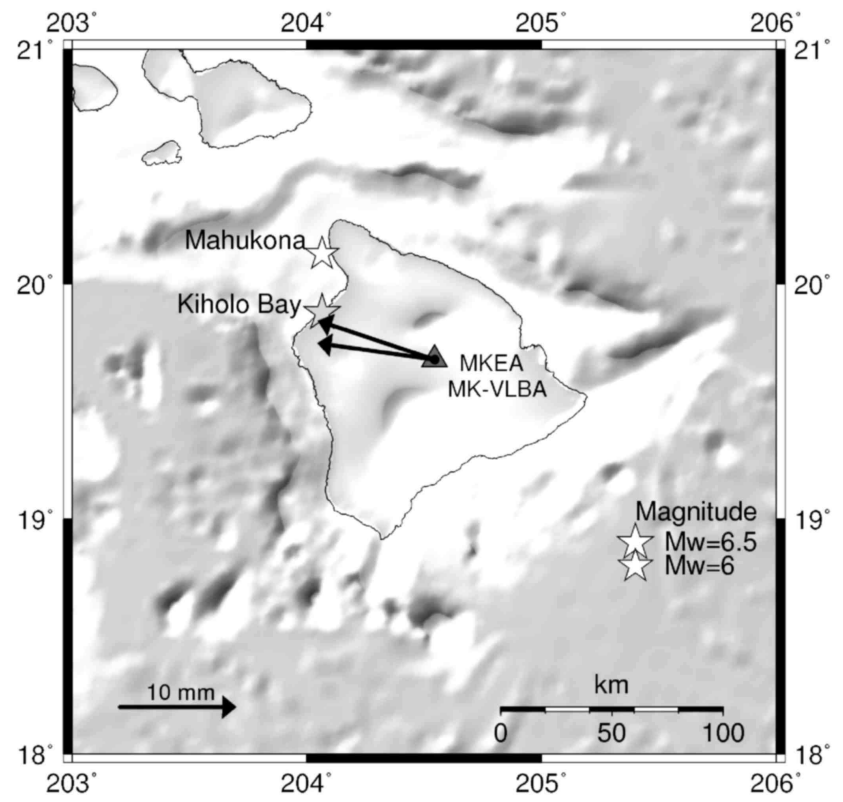

Fig. 8 Map of the Hawaii Island. Locations of seismic events on 2006.10.15 are shown with stars. The arrows show the MK-VLBA site displacement caused by these events from analysis of VLBI observations (bottom) and GPS observations (above).

\subsection{Detection of post-seismic deformations}

One possible cause of non-linear site motions is seismic events. These events are recorded by networks of seismology instruments and their analysis allows the derivation of additional information, such as timing of the event, its magnitude, or direction of a slip. Such information is independent of geodesy measurements and can be used for verification of our VLBI results.

On 2006.10.15 two powerful earthquakes struck the Island of Hawaii. A magnitude 6.7 event occurred at 17:07:48 UTC and was located $16 \mathrm{~km}$ north-west of Kailua Kona, a town on the west coast of the Big Island $\left(19^{\circ} .820 \mathrm{~N}, 156^{\circ} .027 \mathrm{~W}\right)$ in the Kiholo Bay, 38 $\mathrm{km}$ beneath the surface. The Kiholo Bay event was followed by a magnitude 6.0 Makuhona event 7 minutes later, located $44 \mathrm{~km}$ north of the airport and at a $20 \mathrm{~km}$ depth. The epicenters are shown in Figure 4.6. Although the two major events were only 7 minutes apart, their depth difference and aftershock epicenters suggest that the second event may not have been an aftershock of the larger event, and that they had different sources. There were no reported fatalities, but electric power was lost statewide shortly after the event. Despite their moderate depth, the earthquakes generated high accelerations in the epicentral region, with strong ground motions lasting for approximately $20 \mathrm{~s}$ during the Kiholo Bay event, and $15 \mathrm{~s}$ during the Mahuhona event. One station northeast of the epicenter recorded a maximum horizontal acceleration of $1.03 \mathrm{~g}$. 
The largest historical earthquakes in Hawaii have occurred beneath the flanks of Kilauea, Mauna Loa, and Hualalai volcanoes, when stored compressive stresses from magma intrusions into their adjacent rift zones were released. Their sources are related to near-horizontal basal decollements at approximately $10 \mathrm{~km}$ depth, which separate the emplaced volcanic material from the older oceanic crust. In contrast, the Kiholo Bay event was considered tectonic, rather than volcano related. Deeper, 30-40 km deep, earthquakes like this result from a longterm geologic response to flexural fracture of the underlying lithosphere from the load of the island mass (Chock 2006). They are the result long term accumulation and release of lithospheric flexural stresses caused by the island-building process.

The distance from the epicenter of the Kailua Kona event to the MK-VLBA station is $62 \mathrm{~km}$. No structural damage was reported. As a response to the event, an additional observing session was added to the schedule on 2006.11.08 in order to detect possible post-seismic deformation. Preliminary analysis early in 2007 did not find any position changes exceeding $1 \mathrm{~cm}$.

We reanalyzed the dataset and parameterized nonlinear motion with the B-spline of the zeroth degree with two knots: at 1994.07.08 (beginning observations) and 2006.10.15 17:07:48. Using these two estimates of B-spline coefficients and their covariance matrices, we computed the displacement vector:

$$
\begin{array}{rr}
\mathrm{Up}= & -7.7 \pm 1.3 \mathrm{~mm} \\
\text { East } & =-10.0 \pm 0.4 \mathrm{~mm} \\
\text { North } & =1.5 \pm 0.4 \mathrm{~mm}
\end{array}
$$

where the quoted uncertainties are unscaled 1- $\sigma$ formal errors. The vertical and west displacements look very significant. However, the presence of outliers may cause an artificial jump and various systematic correlated errors may cause estimates of uncertainties to be unreliable.

In order to check the robustness of the solution, we performed two tests: an observation decimation test and a knot shift test. In the observation decimation test we ran two solutions. The first solution used only the odd observations, while the second solution used only the even observations. The data used in these solutions are independent. This test checks the contribution of random errors uncorrelated at time scale of the interval between observations, typically $5-10$ minutes. The difference in estimates of the displacement was only $0.14 \mathrm{~mm}$ in the vertical component and $0.06 \mathrm{~mm}$ in the horizontal component.

In the knot shift test we made 21 trial solutions which differed only by epochs of the B-spline knots. In each trial solution we shifted the epoch of the knot six months backward with respect to the previous. The procedure for computing theoretical path delay for these trial solutions incorporated the estimate of the displacement at 2006.10.15 17:07:48 epoch from the initial solution. The rms of the time series of displacement estimates at epochs with no reported seismic events were $2.1 \mathrm{~mm}$ for the vertical and $1.3 \mathrm{~mm}$ for the horizontal component of the MK-VLBA displacement vector. We consider these statistics as a measure of the robustness of the estimates of the displacement vector. Both tests support our claim that VLBI observations detected a displacement of station MK-VLBA caused by the seismic event at 2006.10.15 at the confidence level of 99.5\%.

Since there is a GPS receiver named MKEA located 88 meters from the VLBA station MK-VLBA, we examined the GPS site motion series to determine the corresponding co-seismic offset. For the analysis, we used the MKEA daily position time series generated by JPL (M. Heflin, 2007, personal communication). We obtained the following estimate for the co-seismic displacement vector:

$$
\begin{aligned}
\mathrm{Up} & =-6.3 \pm 0.9 \mathrm{~mm} \\
\text { East } & =-9.9 \pm 0.4 \mathrm{~mm} \\
\text { North } & =3.5 \pm 0.2 \mathrm{~mm}
\end{aligned}
$$

Here, the uncertainties are unscaled 1-sigma formal errors. This is reasonable because the position repeatabilities computed from the position time series are close to the formal uncertainties of the daily estimates. The VLBI and GPS vertical and east displacements are consistent within their 1-sigma error bars but the $2 \mathrm{~mm}$ difference in the north displacement is too large to be explained by the formal uncertainties.

\subsection{Harmonic variations in site positions}

The technique of estimation of harmonic variations in site positions directly from the analysis of group delays was developed by Petrov and Ma (2003). It was shown in that study that many stations exhibit position variations that are attributed to mismodeled harmonic nontidal signals. The purpose of estimating the harmonic site position variations was to remove those remaining signals. We estimated sine and cosine amplitudes of variations in all three components of site position vectors at annual (Sa), semi-annual (SSa), diurnal $\left(S_{1}\right)$, and semi-diurnal $\left(S_{2}\right)$ frequencies for all VLBA and 25 other non-VLBA stations. The seasonal signal is caused by unaccounted hydrology loading, by errors in annual amplitudes of the NMF mapping function that lead to systematic errors in tropospheric path delay modeling, and possibly other effects. Sun-synchronous diurnal 
Table 7 Amplitudes of vertical component of harmonic variations of VLBA station positions in $\mathrm{mm}$.

\begin{tabular}{lclcl}
\hline Station & annual & semi-annual & diurnal & semi-diurnal \\
\hline BR-VLBA & $8.0 \pm 0.3$ & $3.3 \pm 0.3$ & $1.0 \pm 0.2$ & $0.5 \pm 0.2$ \\
FD-VLBA & $1.8 \pm 0.3$ & $1.8 \pm 0.3$ & $0.4 \pm 0.2$ & $1.5 \pm 0.2$ \\
HN-VLBA & $5.4 \pm 0.3$ & $2.3 \pm 0.3$ & $1.5 \pm 0.3$ & $1.0 \pm 0.3$ \\
KP-VLBA & $1.7 \pm 0.3$ & $1.8 \pm 0.3$ & $0.2 \pm 0.2$ & $0.8 \pm 0.2$ \\
LA-VLBA & $1.0 \pm 0.3$ & $3.2 \pm 0.2$ & $1.3 \pm 0.2$ & $0.9 \pm 0.2$ \\
MK-VLBA & $2.5 \pm 0.4$ & $2.5 \pm 0.4$ & $0.8 \pm 0.3$ & $2.5 \pm 0.3$ \\
NL-VLBA & $4.1 \pm 0.3$ & $3.6 \pm 0.3$ & $0.8 \pm 0.2$ & $0.2 \pm 0.2$ \\
OV-VLBA & $2.7 \pm 0.3$ & $2.0 \pm 0.3$ & $1.1 \pm 0.2$ & $0.9 \pm 0.2$ \\
PIETOWN & $1.8 \pm 0.3$ & $0.8 \pm 0.3$ & $1.1 \pm 0.2$ & $1.2 \pm 0.2$ \\
SC-VLBA & $2.6 \pm 0.5$ & $3.0 \pm 0.5$ & $0.5 \pm 0.4$ & $1.3 \pm 0.4$ \\
\hline
\end{tabular}

Table 8 Amplitudes of horizontal component of harmonic variations of VLBA station positions in $\mathrm{mm}$.

\begin{tabular}{lcccc}
\hline Station & annual & semi-annual & diurnal & semi-diurnal \\
\hline BR-VLBA & $0.8 \pm 0.1$ & $0.3 \pm 0.1$ & $0.3 \pm 0.1$ & $0.2 \pm 0.1$ \\
FD-VLBA & $1.4 \pm 0.1$ & $0.1 \pm 0.1$ & $0.2 \pm 0.1$ & $0.1 \pm 0.1$ \\
HN-VLBA & $0.9 \pm 0.1$ & $0.2 \pm 0.1$ & $0.2 \pm 0.1$ & $0.1 \pm 0.1$ \\
KP-VLBA & $1.5 \pm 0.1$ & $0.3 \pm 0.1$ & $0.4 \pm 0.1$ & $0.2 \pm 0.1$ \\
LA-VLBA & $1.1 \pm 0.1$ & $0.3 \pm 0.1$ & $0.3 \pm 0.1$ & $0.1 \pm 0.1$ \\
MK-VLBA & $0.8 \pm 0.2$ & $0.4 \pm 0.2$ & $0.8 \pm 0.1$ & $0.3 \pm 0.1$ \\
NL-VLBA & $0.7 \pm 0.1$ & $0.2 \pm 0.1$ & $0.2 \pm 0.1$ & $0.2 \pm 0.1$ \\
OV-VLBA & $1.2 \pm 0.1$ & $0.2 \pm 0.1$ & $0.5 \pm 0.1$ & $0.3 \pm 0.1$ \\
PIETOWN & $1.5 \pm 0.1$ & $0.2 \pm 0.1$ & $0.3 \pm 0.1$ & $0.3 \pm 0.1$ \\
SC-VLBA & $0.7 \pm 0.2$ & $0.9 \pm 0.2$ & $0.4 \pm 0.1$ & $0.3 \pm 0.1$ \\
\hline
\end{tabular}

variations can be caused by thermal variations, by systematic errors in tropospheric path delay, or unmodeled non-tidal ocean loading.

In order to evaluate the robustness of the estimates at low frequencies, we performed two tests: the observation decimation test and the dummy frequency test. We examined the differences in estimates of sine and cosine amplitudes from the observation decimation test and compared them with the formal uncertainties of the estimates. The differences are within 1-sigma formal uncertainty.

In the second test we estimated site position variations at a frequency of $2.5 \cdot 10^{-7} \mathrm{rad} \mathrm{s}^{-1}$, corresponding to a period of 0.8 year where no harmonic signal is expected. The average amplitude found for the vertical displacements for the ten VLBA stations is $1.2 \mathrm{~mm}$, and $0.2 \mathrm{~mm}$ for the horizontal displacements. These estimates should be considered as the upper limit of uncertainties, since the observed harmonic signal at frequency $2.5 \cdot 10^{-7} \mathrm{rad} \mathrm{s}^{-1}$ is affected by both systematic errors and real displacements at this frequency, caused by anharmonic, broad-band site position displacements.

We see that the combined contribution of seasonal position variation, unaccounted for in the theoretical model, can reach $1 \mathrm{~cm}$ for the vertical component of VLBA stations and $1.5 \mathrm{~mm}$ for the horizontal component and is statistically significant for most of the sta- tions at the $95 \%$ confidence level. Unaccounted diurnal position variations are at the level of 1-2 mm.

\section{Error analysis}

Uncertainties of estimated parameters can be evaluated using the law of error propagation under the assumption that the unmodeled contribution to group delay is due to random uncorrelated errors with known variance. The parameter estimation procedure provides estimates of these errors based on the SNR of fringe amplitudes. These errors are labeled as formal errors and they are considered as lower limits of accuracy. Formal uncertainties of the site position estimates of the VLBA stations from our global solution are in the range of $0.5-1.0 \mathrm{~mm}$ for vertical components and 0.2 $0.5 \mathrm{~mm}$ for horizontal components. Formal uncertainties of the VLBA site velocity estimates are in the range of $0.07-0.1 \mathrm{~mm} / \mathrm{yr}$ for vertical components and 0.04 $0.05 \mathrm{~mm} / \mathrm{yr}$ for horizontal components.

Many factors contribute to an increase of errors. Among them are underestimated uncertainties of group delays due to phase instability of the data acquisition system, unmodeled instrumental errors, unaccounted atmospheric fluctuations, correlations between observations, and unaccounted environmental effects.

Another measure of accuracy is an observation decimation test. Since the two datasets have independent random errors, the root mean square of differences between estimates from these solutions divided by $\sqrt{2}$ provides a measure of accuracy that is independent of estimates of the uncertainty of each individual observation.

However, many other factors that affect the results, such as mismodeled delay in the neutral atmosphere, are common in the two subsets. To examine the influence of these factors, we ran a session decimation test and used every second observing session. In the observation decimation test, matrices of observation equations were almost identical, but the data were affected by the same systematic errors. In the session decimation test, systematic errors were more independent, but the matrices of observation equations have larger differences.

The statistics of differences are given in Table 9. In the absence of systematic errors, both decimation tests would give close results. Analysis of the statistics shows significant discrepancies between the decimation tests. Estimates of site positions and velocities in solutions where every second observation is removed are a factor of 2-3 closer to each other than in solutions where every second session is removed. This is an indication that systematic errors on the time scale of several minutes - the typical time between observations - are 
Table 9 Formal uncertainties and rms of differences of two decimation tests for estimates of site positions and site velocities. The estimates are given for horizontal and vertical components separately.

\begin{tabular}{lcccc}
\hline Statistics & \multicolumn{2}{c}{ Position } & \multicolumn{2}{c}{ Velocity } \\
& \multicolumn{2}{c}{$\mathrm{mm}$} & \multicolumn{2}{c}{$\mathrm{mm} / \mathrm{yr}$} \\
& $\mathrm{v}$ & $\mathrm{h}$ & $\mathrm{v}$ & $\mathrm{h}$ \\
\hline Formal $\sigma$ & 0.7 & 0.3 & 0.11 & 0.04 \\
Observation decimation & 0.3 & 0.06 & 0.04 & 0.02 \\
Session decimation & 1.4 & 0.3 & 0.09 & 0.06 \\
\hline
\end{tabular}

Table 10 The rms of differences in pole coordinates estimates between the VLBI results and the GPS time series igs00p03.erp. Only data after 1997.0 are used. Comparison is made separately for VLBA sessions in astrometric mode (only 10 VLBA stations) and VLBA sessions in geodetic mode (10 VLBA stations plus 3-10 non-VLBA stations).

\begin{tabular}{lll}
\hline Sessions & X-pole & Y-pole \\
\hline VLBA, Astrometric mode & $0.87 \mathrm{nrad}$ & $1.15 \mathrm{nrad}$ \\
VLBA, Geodetic mode & $0.54 \mathrm{nrad}$ & $0.43 \mathrm{nrad}$ \\
IVS sessions in 2006-2007 & $0.39 \mathrm{nrad}$ & $0.47 \mathrm{nrad}$ \\
\hline
\end{tabular}

correlated. The session decimation test suggests that estimates of the vertical site position errors should be scaled by a factor of 2 . This scaling may be related to unaccounted errors in modeling the contribution of the neutral atmosphere.

We also estimated Earth orientation parameters in our solutions. Comparison of our EOP estimates with independent GPS time series igs00p03.erp ${ }^{5}$ gives us another measure of the accuracy of our results. We computed the rms of differences in pole coordinates for sessions in astrometric mode and sessions in geodetic modes. Only sessions after 1997 were used for this comparison, since GPS estimates prior to this date are not very accurate. As we see from Table 10, the VLBA estimates of pole coordinates from geodetic observations are approximately as close to GPS results as ones from regular IVS sessions. However, the EOP from astrometric sessions divert from the GPS time series by a factor of 2 larger than the EOP from geodetic VLBI sessions.

A baseline length repeatability test provides another measure of solution accuracy. For each baseline, a series of lengths was obtained. Empirical non-linear site position variations described above were applied as a priori. A plot of the baseline length repeatability of VLBA baselines is presented in Figure 5. For comparison, baseline length repeatability at non-VLBA baselines is also shown.

A linear model of baseline lengths was fit to each series, and the wrms of the deviations from the linear model, the baseline length repeatability, was computed for each baseline. The plot of baseline length repeatabil-

5 Available at

ftp://cddisa.gsfc.nasa.gov/gps/products/igs00p03.erp.Z

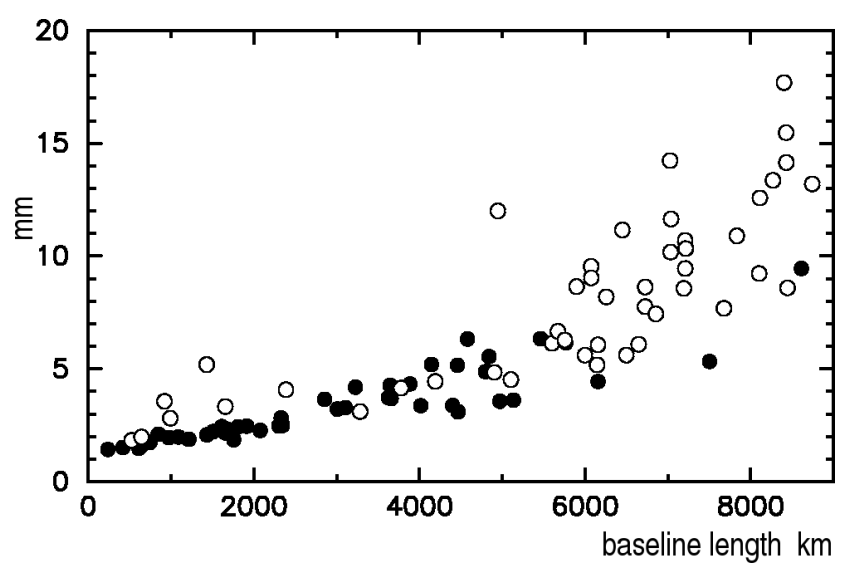

Fig. 9 Baseline length repeatability as a function of baseline length. Solid disks shows estimates of baseline length repeatability between VLBA sites, circles shows repeatability between non-VLBA sites.

ity shows that the scatter in baseline lengths estimates between VLBA sites is less than the scatter in baseline length between dedicated geodetic VLBI stations. The set of wrms was fit by a function $\sqrt{A^{2}+(B \cdot L)^{2}}$ where $L$ is the mean baseline length. Coefficients $A$ and $B$, which represent the average baseline length repeatability, are a measure of accuracy. For the VLBA baselines, $\mathrm{A}=1.6 \mathrm{~mm}, \mathrm{~B}=0.9 \mathrm{ppb}$, for non-VLBA baselines $\mathrm{A}=2.0 \mathrm{~mm}, \mathrm{~B}=1.4 \mathrm{ppb}$. Growth of the baseline length repeatability with the baseline length for both sets of data reflects the impact of the contribution of unmodeled path delay in the neutral atmosphere, which affects the site position vertical component to a greater extent than the horizontal one (Davis et al. 1985).

The results of error analysis allow us to conclude that the errors of predicted site positions for any epoch within the time range of observations, [1994, 2008], are in the range of $2-3 \mathrm{~mm}$ for the vertical component and $0.4-0.6 \mathrm{~mm}$ for the horizontal component. The predicted positions, based on the adjusted parameters of the site motion model, includes mean site positions at the reference epoch, site velocities, and coefficients of the harmonic and B-spline models. The estimates of errors for the vertical coordinates were derived from the formal errors by inflating by a factor of 2 , as the session decimation test suggests. In the absence of new observations, the predicted errors in site position will grow by a factor of 2 by 2020 as is shown in Figure 5, provided no motion other than harmonic position variations and linear velocities, i.e. that no seismic events, or unmodeled variable tilt, will happen in the future. 


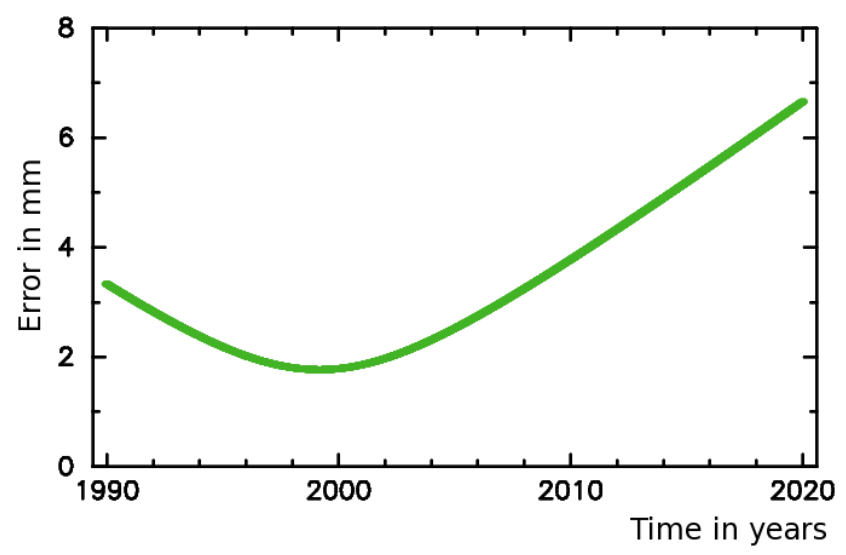

Fig. 10 Predicted uncertainties of MK-VLBA vertical coordinate if no additional data are taken after 2007. The errors were inflated by a factor of 2 with respect to the formal errors. The uncertianties of positions of other stations have a similar growth.

\section{Conclusions}

The observing campaign for monitoring positions of the VLBA sites during 1994-2007 has been highly successful. From analysis of 14 years of data, the elements of the VLBA array during that period were determined with an accuracy of $2-3 \mathrm{~mm}$ in the vertical and $0.4-$ $0.6 \mathrm{~mm}$ in the horizontal component. This meets the requirements for position accuracy for astrometry and astrophysics programs at the VLBA.

The baseline length repeatability between VLBA stations is smaller than that for non-VLBA IVS stations. EOP estimates from geodetic VLBA sessions are as close to GPS results as EOP estimates from the IVS sessions dedicated to precise EOP determination.

We found that the positions of all VLBA stations exhibit a significant seasonal signal with amplitudes of $1-8 \mathrm{~mm}$ in the vertical and $0.5-3.5 \mathrm{~mm}$ in the horizontal component.

Several stations show anharmonic signals in their positions. We have traced the origin of these signals to co-seismic deformations (MK-VLBA) and to a timevarying antenna tilt (PIETOWN). In the case of tilt, the signal can be successfully modeled using the pointing adjustment model, however, the scaling factor between the antenna tilt and motion of the antenna reference point has to be determined from VLBI observations.

We derived an empirical model of site motion that consists of linear velocity, a set of coefficients of the harmonic expansion, and coefficients of the B-spline model that takes into account ad hoc motions. For the case where no ad hoc motion occurs in the future, the accuracy of VLBA station position predictions would gradually degrade to $5-8 \mathrm{~mm}$ in the vertical and $1-$ $1.5 \mathrm{~mm}$ in the horizontal by the year 2020 in the absence of future observations. However, unpredictable events, such as local deformations or post-seismic deformation could cause significantly larger errors. Therefore, continuation of VLBA site position monitoring is highly desirable.

Acknowledgements The National Radio Astronomy Observatory is a facility of the National Science Foundation operated under cooperative agreement by Associated Universities, Inc. We thank the staff of the VLBA for carrying out and correlating these observations in their usual efficient manner. We also thank M. Titus and B. Corey from Haystack Observatory for their efforts in re-correlation of the rdv22 data on the Haystack correlator. Lastly, we are thankful to A. Nothnagel and A. Niell for valuable comments that helped to improve this manuscript. This work was done while J. Gipson, D. Gordon, D. MacMillan and L. Petrov worked for NVI, Inc. under NASA contract NAS5-01127.

\section{References}

Agnew, DC (1997) NLOADF: A program for computing oceantide loading, J Geophys Res, 102(B3):5109-5110

Beasley AJ, Gordron D, Peck AB, Petrov L, MacMillan DS, Fomalont EB, Ma C (2002) The VLBA calibrator survey VCS1', Asrophys J Supp, 141:13-21, doi:10.1086/339806

Benson J (1995) The VLBA Correlator, in Zensus JA, Diamond PJ, Napier PJ, Proceedings from the 1993 NRAO Summer School, ASP Conference Series, 82, 113-131 http://www.cv.nrao.edu/vlbabook

De Boor C (1978) A practical guide to splines, Springer-Verlag, New York.

Chock G (2006) Preliminary observations on the Hawaii earthquakes of October 15, 2006, EERI special earthquake report.

Davis JL, Herring TA, Shapiro II, Rogers AEE, Elgered G (1985) Geodesy by radio interferometry: Effects of atmospheric modeling errors, Radio Sci, 20(6):1593-1607

Hawarey M, Hobiger T, Schuh H (2005) Effects of the 2nd order ionospheric terms on VLBI measurements, Geophys Res Let, 32:L11304, doi:10.1029/2005GL022729

Hinteregger HF, Shapiro II, Robertson DC, Knight CA, Ergas RA, Whitney AA, Rogers AEE, Moran JM, Clark TA, Burke BF (1972) Precision Geodesy via Radio Interferometry, Science, 178:396-398, doi:10.1126/science.178.4059.396

Greisen EW (2003) Processing and archiving of AIPS, the VLA, and the VLBA, In Information handling in astronomy - Historical vistas, Heck A ed., Kluwer Academic Publishers, Dordrecht, ISBN 1-4040-1178-4, Astrophys and Space Sci Lib, 285, 109 ( Available at http://www.aoc.nrao.edu/ egreisen/aips.ps.gz )

Kalnay EM, Kanamitsu M, Kistler R, Collins W, Deaven D, Gandin L, Iredell M, Saha S, White G, Woollen J, Zhu Y, Leetma A, Reynolds R, Chelliah M, Ebisuzaki W, Higgins W, Janowiak J, Mo KC, Ropelewski C, Wang J, Jenne R, Joseph D (1996) The NCEP/NCAR 40-year reanalysis project, Bull Amer Meteorol Soc, 77:437-471

Kopeikin SM, Schäfer G (1999) Lorentz covariant theory of light propagation in gravitational fields of arbitrary-moving bodies, Phys Rev D, 60(12):124002, doi:10.1103/PhysRevD.60.124002

Kovalev YY, Lobanov AP, Pushkarev AB, \& Zensus JA (2008) Opacity in compact extragalactic radio sources and its effect on astrophysical and astrometric studies, Astron \& Astrophys, 483(3):759-768, doi:10.1051/0004-6361:20078679

Mathews PM (2001) Love numbers and gravimetric factors for diurnal tides, J Geod Soc Japan, 47(1):231-236 
Matsumoto K, Takanezawa T, Ooe M (2000) Ocean tide models developed by assimilating TOPEX/POSEIDON altimeter data into hydrodynamical model: a global model and a regional model around Japan, J Oceanography, 56:567-581

Matveenko LI, Kardashev NS, \& Sholomitsky GB (1965) On an interferometer with very long baseline, Izvestija Vysshih Uchebnyh Zavedenij, Radiofizika, 8:651-654 (In Russian). English translation: Large base-line radio interferometers, Radiophysics and Quantum Electronics, 8(4): 461-463, 1965, doi: $10.1007 / \mathrm{BF} 01038318$

McCarthy DD and Petit G, Eds (2004) IERS Conventions 2003 IERS Technical Note, No. 32, Frankfurt am Main: Verlag des Bundesamts für Kartographie und Geodäsie

Napier PJ, Bagri DS, Clark BG, Rogers AEE, Romney JD, Thompson AR, Walker RC (1994) The Very Long Baseline Array, Proc IEEE, 82(5):658-671

Niell AE, (1996) Global mapping functions for the atmosphere delay at radio wavelengths, J Geophys Res, 100:3227-3246

Nothnagel A, Bromorzki O, Campbell J, Muskens A, Rottmann H, Rottman I (2002) Comparison of the output of repeated Mark III and Mark IV correlation results. In IVS 2002 general meeting proceedings, ed Vandenberg N \& Baver KD, 107-112

Nürnberger G (1989) Approximation by spline functions, Springer, New York.

Petrov L and Ma C (2003), Study of harmonic site position variations determined by VLBI, J Geophys Res, 108(B4), 2190, doi: 10.1029/2002JB001801

Petrov L and Boy JP (2004) Study of the atmospheric pressure loading signal in VLBI observations, J Geophys Res, 109:B03405, doi:10.1029/2003JB002500

Petrov L (2007) The empirical Earth rotation model from VLBI observations, Astron \& Astrophys, 467(1):359-369, doi:10.1051/0004-6361:20065091

Piner BG, Mahmud M, Fey AL, Gospodinova K (2007) Relativistic jets in the radio reference frame image database. I. Apparent Speeds from the First 5 Years of Data, Astron J, 133(5):2357-2388, doi:10.1086/514812

Ray RD (1999) A global ocean tide model from TOPEX/ POSEIDON altimetry: GOT99.2, NASA/TM-1999-209478, Greenbelt USA

Ray J and Altamimi Z (2005) Evaluation of co-location ties relating the VLBI and GPS reference frames, J. Geod, 79:189-185, doi:10.1007/s00190-005-0456-z

Saastamoinen J (1972a) Contributions to the theory of atmospheric refraction, Bull Geod, 105:279-298

Saastamoinen J (1972b) Introduction to practical computation of astronomical refraction. Part II Bull Geod, 106:383-397

Shapiro II, Knight CA (1970) Geophysical applications of longbaseline radio interferometry. In Earthquake displacement field and the rotation of the Earth, ed. Beck A, Reidel, Dordecht, 284

Schlüter W, Behrend D (2007) The international VLBI service for geodesy and astrometry (IVS): current capabilities and future prospects, J Geod, 81(4-5):379-387, doi:10.1007/s00190-0060131-z

Sovers OJ, Fanselow JL, Jacobs CS (1998) Astrometry and geodesy with radio interferometry: experiments, models, results, Rev Modern Phys, 70(4):1393-1454, doi:10.1103/RevModPhys.70.1393

Steigenberger P, Rothacher M, Dietrich R, Fritsche M, A. Rülke, Vey S (2006): Reprocessing of a global GPS network. J Geophys R, 111, B05402, doi: 10.1029/2005JB003747

Takahashi F, Kondo R, Takahashi Y, Koyama Y (2000) Very long baseline interferometer, Ohmsha, Ltd, Tokyo
Thompson AR, Moran JM, Swenson GW (2001) Interferometry and synthesis in radio astronomy, Wiley \& Sons, Inc.

Walker RC (1999) Pointing improvements using rail height information, VLBA test memo 61, National Radio Astronomy Observatory, Socorro, USA http://www.vlba.nrao.edu/memos/test/test61memo.ps

Walker RC (1995) What the VLBA Can Do For You, in Zensus JA, Diamond PJ, Napier PJ, Proceedings from the 1993 NRAO Summer School, ASP Conference Series, 82, 133-157 http://www.cv.nrao.edu/vlbabook

Whitney AR, Cappallo R, Aldrich W, Anderson B, Bos A, Casse J, Goodman J, Parsley S, Pogrebenko S, Schilizzi R \& Smythe D (2004) Mark 4 VLBI Correlator: Architecture and algorithms, Radio Sci, 39, RS1007 doi: $10.1029 / 2002 R S 002820$ 


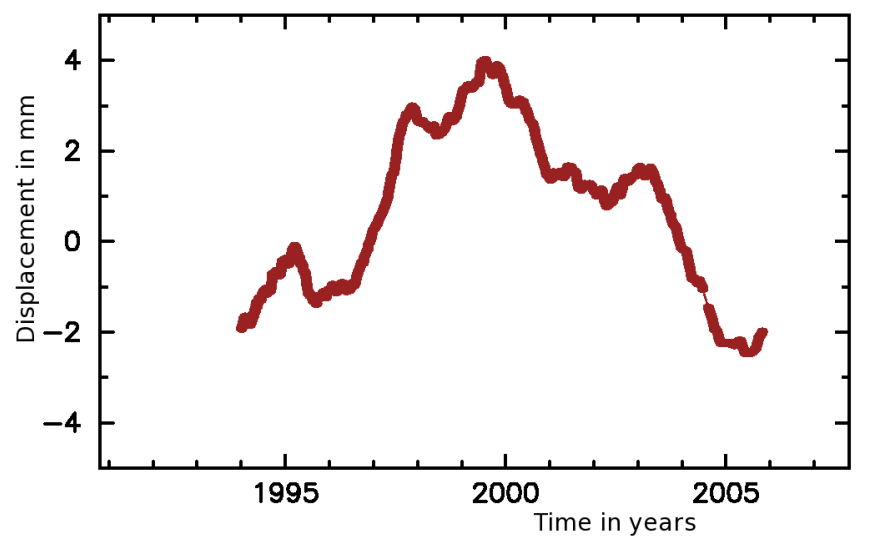




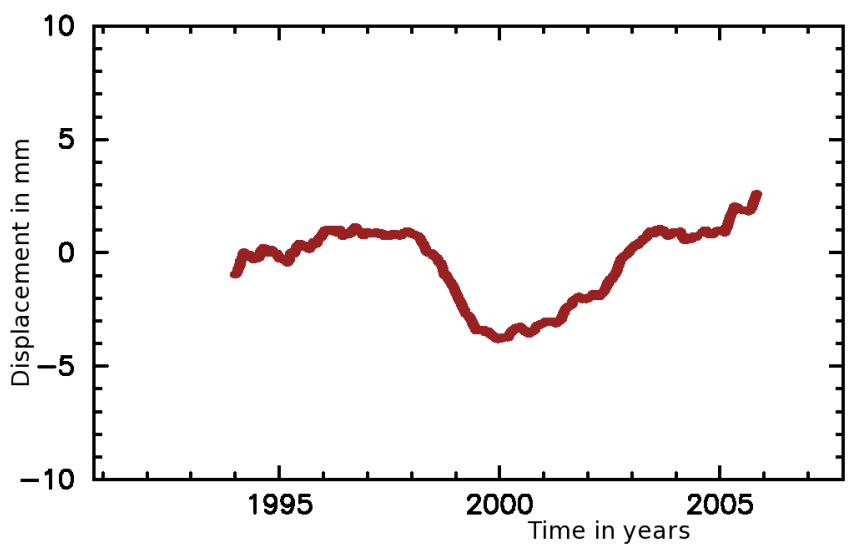

